\title{
Exploración lexicográfica de seis diccionarios del maya yucateco
}

Lexicographic exploration of six Yucatec Maya dictionaries

Catherine Rhodes Universidad de Nuevo México, Departamento de Antropología Irma Yolanda Pomol Cahum Universidad de Oriente, Licenciatura en Lingüística y Cultura Maya Miguel Óscar Chan Dzul Universidad de Oriente, Licenciatura en Lingüística y Cultura Maya

\section{Resumen}

Este artículo es una investigación exploratoria sobre las características de seis diccionarios en maya yucateco, seleccionados a partir de una encuesta en línea a usuarios, hablantes nativos y no-nativos de la lengua maya: Academia de la Lengua Maya de Yucatán (ALmy, 2003), Bastarrachea Manzano, Yah Pech \& Briceño Chel (1992), Bricker, Po'ot Yah \& Dzul de Po'ot (1998), Gómez Navarrete (2009), Martínez Huchim (2008) y Montgomery (2004). Se desarrolló una rúbrica para la evaluación de los diccionarios, según Fuentes Morán, García \& Torres (2005) y recomendaciones sobre la creación de diccionarios en lenguas indígenas (Callaghan, 2002; Frawley, Hill \& Munro, 2002; Hinton \& Weigel, 2002), así como monolingües y bilingües. Se analizó la macroestructura (organización de la obra, extensión, ortografía y alfabeto) y microestructura de los diccionarios (especificación gramatical, glosas, ejemplos, variantes y préstamos léxicos); también se discutieron estas características desde las perspectivas de los usuarios encuestados. Finalmente, se presentan recomendaciones respecto a las ideologías lingüísticas en la elaboración de diccionarios, antes de sugerir vías para futuras investigaciones.

Palabras clave: diccionario bilingüe; evaluación de diccionarios; purismo lingüístico; ideologías lingüísticas 


\begin{abstract}
This article is an exploratory research study of the characteristics of six Yucatec Maya dictionaries, selected based on a web survey of users who are native and non-native speakers of the Maya language: Academia de la Lengua Maya de Yucatán (ALmy, 2003), Bastarrachea Manzano, Yah Pech \& Briceño Chel (1992), Bricker, Po'ot Yah \& Dzul de Po'ot (1998), Gómez Navarrete (2009), Martínez Huchim (2008) and Montgomery (2004). We developed a rubric for evaluating the dictionaries, following Fuentes Morán, García $\&$ Torres (2005) and the recommendations on the elaboration of monolingual and bilingual dictionaries in Indigenous lan-

guages (Callaghan, 2002; Frawley, Hill \& Munro, 2002; Hinton \& Weigel, 2002). The analysis of the dictionaries was conducted based on macrostructure (text organization, text extension, orthography, and alphabet) and microstructure of the dictionaires (grammatical specification, glosses, examples, variants, and lexical loans); these characteristics are also discussed from the perspectives of the users surveyed. Finally, we include our recommendations regarding the role of linguistic ideologies in the elaboration of dictionaries, before suggesting lines for future research.
\end{abstract}

Keywords: bilingual dictionary; dictionary evaluation; linguistic purism; linguistic ideologies

Fecha de recepción del artículo: 6 de marzo de 2017 Fecha de recepción de la versión revisada: 9 de mayo de 2018 Fecha de aceptación: 22 de mayo de 2018 


\section{Introducción}

Desde las últimas tres décadas han proliferado textos de consulta en lengua maya, principalmente, diccionarios bilingües de la lengua. Estos textos varían ampliamente en su calidad, metodología, elección de léxico, contenido y destinatario. Por esta razón, evaluamos textos de referencia contemporáneos como una herramienta para que los usuarios puedan contar con información que les permita guiar su consulta de estos materiales. Con el fin de priorizar la utilidad de los resultados de este trabajo, analizamos los diccionarios más utilizados por hablantes nativos, de segunda lengua, profesores o aprendices de la lengua maya. Reconocemos de antemano que, aunque esta investigación es lexicográfica, nosotros no somos ni pretendemos ser lexicógrafos. Esta es una evaluación basada tanto en las mejores prácticas propuestas por la literatura como en las opiniones y preferencias de los usuarios de estos diccionarios obtenidas por medio de una encuesta. También señalamos que este trabajo es exploratorio y que el tema requiere de futuras investigaciones.

Inicialmente, discutimos en $\$ 2$ los criterios para evaluar diccionarios según el marco evaluativo planteado por Fuentes Morán, García Palacios y Torres del Rey (2005). En esta sección se describe cómo elegimos la muestra de entradas evaluada en cada diccionario. En $\S 3$ explicamos la selección de los diccionarios a evaluar. Posteriormente, en $\S 4$ ofrecemos una discusión del análisis de los diccionarios, en el cual se consideraron sus características generales. Finalmente, en $\$ 5$ presentamos las conclusiones y una serie de sugerencias y vías para futuras investigaciones.

\section{Evaluación de diccionarios}

\subsection{Rúbrica para la evaluación de diccionarios según Fuentes Morán, García Palacios y Torres del Rey}

Como primer paso de nuestro estudio, revisamos la literatura sobre la creación de diccionarios monolingües, bilingües y de lenguas indígenas y resumimos las recomendaciones y mejores prácticas sugeridas. Asimismo, sintetizamos una lista de elementos de evaluación y creamos una rúbrica para el mismo fin (véa- 
se Cuadro 1), basada en las recomendaciones de Fuentes et al. (2005) y de otros textos afines: Bartholomew y Schoenhals (1983), Haensch y Omeñaca (2004) y Piotrowski (1994).

Cuadro 1. Rúbrica para la evaluación de diccionarios según Fuentes Morán, García Palacios y Torres del Rey (2005)

1. ¿Ofrece el diccionario suficientes datos?

2. ¿Se puede encontrar fácil y rápidamente la información que se busca y que el diccionario contiene?

3. ¿Son los datos inteligibles?

4. ¿Permite el diccionario valorar su fiabilidad?

\subsubsection{Criterios sobre la cantidad de datos}

Fuentes et al. (2005) señalan que los diccionarios deben ser susceptibles a un análisis que permita a los usuarios determinar el grado de confianza que en ellos se deposita, dado que frecuentemente se sitúan como un eje del engranaje informativo para sus usuarios. Estos autores indican que uno de los puntos más importantes en la evaluación de un diccionario es la suficiencia de datos; sin embargo, enfatizan que esto no debe basarse únicamente en "el número de entradas", ya que estas "no son fácilmente delimitables porque no son fácilmente objetivables" (Fuentes et al., 2005: 75). Para la evaluación, se aconseja hacer preguntas como: "¿ofrece datos sobre el tipo de texto en el que puede encontrarse la unidad léxica en cuestión? (es decir, texto especializado frente a texto no especializado), ¿ofrece datos sobre la proyección sintáctica de la unidad léxica en cuestión? O, ¿resuelve dudas sobre las implicaciones pragmáticas del uso de esta unidad?" (Fuentes et al., 2005: 76).

Al referirse a la creación de diccionarios bilingües de lenguas indígenas en particular, Bartholomew y Schoenhals (1983) recomiendan que el diccionario debe ser una muestra representativa de lo que el/la lingüista aprende sobre los recursos léxicos de la lengua vernácula durante el transcurso de su trabajo de campo y también de los resultados de investigaciones específicas durante la compilación del diccionario para publicación. ${ }^{1}$ Estos autores hacen una serie de

1 El texto original es: "The finished dictionary should be a representative sample of what the linguist learns about the lexical resources of the vernacular during the course of his field work 
recomendaciones para la inclusión de léxico en el diccionario, mismas que resumimos a continuación (Bartholomew \& Schoenhals, 1983: 21):

- Formar el núcleo del diccionario con estudios de fonología y gramática de la lengua, complementados con palabras adquiridas en contextos cotidianos con los hablantes;

- a este núcleo agregar listas de equivalentes locales del español estándar, sobre todo las más utilizadas en el país en cuestión;

- asegurar que el diccionario refleje el vocabulario de la cultura de los hablantes (e. g., cuando no exista equivalente directo, la entrada puede ser definida o explicada);

- incluir material escrito en la lengua indígena, considerando literatura escrita por nativo hablantes y textos orales;

- cubrir todo el rango de conjunciones, preposiciones y otras palabras básicas para la cohesión del discurso local, y, finalmente,

- reflejar los dominios semánticos en apéndices, así como en el cuerpo del diccionario.

\subsubsection{Criterios sobre accesibilidad a los datos}

Fuentes et al. (2005) indican que para evaluar un diccionario se debe considerar si la información que busca el usuario puede encontrarse fácil y rápidamente y si la obra contiene esa información. Así, "el diccionario debe presentar los datos adecuadamente organizados, es decir, estructurados en vías de acceso que no se interrumpan y con formatos flexibles que no hagan necesario verbalizar qué tipo de información se requiere" (2005: 76). Además, especifican que el diccionario debe contener un sistema de organización de los datos que sea claro y accesible para los usuarios meta - un tema que, argumentan, requiere de más investigación dentro de la lexicografía (Fuentes et al., 2005; véase también Frawley, Hill \& Munro, 2002).

[and] the results of specific investigations conducted during the compilation of the dictionary for publication" (Bartholomew \& Schoenhals, 1983: 21; todas las traducciones en este artículo son nuestras). 


\subsubsection{Criterios sobre inteligibilidad de los datos}

Como indican Fuentes et al., la inteligibilidad de los datos en un diccionario es un punto difícil de evaluar con elementos objetivables, porque esta depende "directamente de mecanismos de comprensión del usuario, de sus procedimientos de adquisición del conocimiento [y] de sus hábitos heurísticos" (2005: 76). Sin embargo, señalan que dicha inteligibilidad se logra si "la definición lexicográfica se basa en una estructura tema/rema que (parcialmente) se corresponde con el binomio información conocida/información desconocida", y esto se puede medir con base en los siguientes puntos:

a) Grado de dificultad de los elementos empleados en la definición y de las posibilidades del usuario de resolver tal grado de dificultad con el propio diccionario de manera ergonómica y satisfactoria.

b) Coherencia y adecuación en la organización de los elementos en las definiciones, teniendo en cuenta factores como los siguientes: ¿cómo percibe la sociedad un determinado concepto?, ¿cómo lo percibe un lingüista u otro tipo de especialista?, ¿qué tipo de percepción se pretende transmitir a través de los datos integrados en el artículo lexicográfico?

c) Coherencia entre los elementos microestructurales externos a la definición con la propia definición, aspecto que se mide en parámetros de redundancia y complementariedad —nunca de contradicción (Fuentes et al., 2005: 77).

\subsubsection{Criterios sobre la fiabilidad del diccionario}

Para la fiabilidad del diccionario, es importante tanto la inteligibilidad del contenido como la calidad de la información provista. Al respecto, Fuentes et al. (2005: 77) señalan que dichos parámetros se miden en gran parte con base en el "prestigio" de quien ha elaborado el diccionario y que para diccionarios bilingües "es importante saber en qué ámbito lingüístico se ha elaborado". Aunado a esto, la editorial que publica el diccionario también resulta un buen indicativo sobre la fiabilidad del texto — uno que ha sido autopublicado, por ejemplo, no necesariamente habrá pasado por un proceso de revisión editorial o de expertos, como habría sido el caso de un texto publicado por una editorial (Monterde Rey, 2007). 
Sin embargo, consideramos altamente subjetivos y culturalmente específicos los conceptos fiabilidad y prestigio.

El segundo punto importante en la evaluación de la fiabilidad de un texto, argumentan Fuentes et al., se plantea a partir de la siguiente pregunta: “ ¿se proporciona datos concretos sobre las fuentes utilizadas tanto para el vaciado de las unidades léxicas que forman parte de la macroestructura como para la obtención del resto de los datos que se proporciona?" (2005: 78). Además, señalan que es importante tener información sobre la actualidad de dichos datos. Asimismo, indican que se acostumbra utilizar "dos tipos de fuentes primarias: datos procedentes de otras fuentes lexicográficas y datos procedentes del vaciado de corpus" - la segunda, establecen, es más prestigiada (2005: 78). Para el desarrollo de diccionarios bilingües de lenguas indígenas, Bartholomew y Schoenhals (1983) enfatizan que el léxico debe provenir de la lengua vernácula. ${ }^{2}$ Por su parte, Callaghan (2002), Frawley et al. (2002) y Hinton y Weigel (2002) señalan incluso que un solo diccionario no necesariamente podrá satisfacer a todos los usuarios.

Bartholomew y Schoenhals (1983) y Haensch (2004) indican que, en la producción de diccionarios bilingües, la fiabilidad del diccionario se basa en la fiabilidad de los equivalentes. Esto se puede medir, en parte, basándose en qué tan acertada es la selección del léxico, lo cual sugiere Haensch se puede averiguar por "la presencia o ausencia de los siguientes tipos de léxico: [...] léxico de nuestra civilización actual sujeto a cambios constantes [...] léxico del lenguaje coloquial actual [...] léxico del lenguaje vulgar [y] americanismos usuales" (2004: 136). ${ }^{3}$ Bartholomew y Schoenhals (1983) establecen que los equivalentes deben basarse en el significado de la palabra en cuestión. Pero, como indica Piotrowski (1994), ningún ítem de la L2 (lengua meta) tiene el mismo significado que el

2 Véase Bartholomew y Schoenhals (1983), capítulo 3, para una explicación completa del proceso que recomiendan.

3 Aunque reconocemos que en algunas comunidades indígenas, sobre todo en los Estados Unidos y Canadá, hay palabras que no se deben incluir por ser consideradas vulgares (Frawley et al., 2002; Hinton \& Weigel, 2002) o secretas (Frawley et al., 2002; Debenport, 2015). Asimismo, varios autores sugieren que el hecho de designar elementos léxicos como coloquiales (o con algún otro término parecido) denota un proyecto no neutral (Frawley et al., 2002; Irvine \& Gal, 2000; Kroskrity, 2002; Rice \& Saxon, 2002). 
equivalente del ítem correspondiente en la L1 (lengua fuente), y que esta diferencia se tiene que asimilar de alguna manera para que la "ecuación" entre las dos lenguas se mantenga verídica. Asimismo, Piotrowski (1994) indica que muchos lingüistas consideran la pragmática más importante que la semántica, ya que las necesidades del usuario pueden determinar la semántica de un diccionario bilingüe, es decir, el tipo de equivalencia.

Finalmente, Fuentes et al. señalan que "la corroboración de los datos que proporciona el diccionario sólo puede hacerse mediante mecanismos externos; los datos del propio diccionario son sólo indicios de su fiabilidad; cuanto mayor es el número de indicios encontrados y estos se presentan de manera más explícita, más fiable podemos considerar el diccionario" (2005: 78). Así, los autores sugieren una lista de "indicios de calidad", que también son indicios "de fiabilidad porque reflejan los métodos de trabajo adoptados en el diccionario", los cuales son:

coherencia formal (por ejemplo, en marcadores estructurales)[;] indicaciones sobre el origen de los datos y sobre los sistemas de procesamiento fines lexicográficos[;] indicaciones sobre la finalidad del diccionario y los destinatarios a los que se orienta[;] postura [explícita] frente a algunos aspectos (por ejemplo socioculturales)[; e] indicios sobre la actualidad de los datos (Fuentes et al., 2005: 78).

Dada esta información, reconocemos que hay criterios generales que pueden ayudar a medir la fiabilidad de un diccionario, pero que también hay consideraciones particulares para diccionarios monolingües — que se basan primordialmente en la fiabilidad de las glosas; breve explicación del significado-, bilingües — que se basan en la fiabilidad de los equivalentes; palabra o expresión correspondiente en otra lengua - y para diccionarios en lenguas indígenas —-sobre todo en el proceso para seleccionar el léxico. ${ }^{4}$

En adición a los elementos elaborados en nuestra rúbrica, consideramos los siguientes puntos para nuestra evaluación de los diccionarios: información que pro-

4 Para más información, véase también Hinton \& Weigel, 2002. 
veen al usuario, información que contienen sobre la gramática de la lengua maya, ortografía y alfabeto que utilizan, trato de préstamos y guía de pronunciación. ${ }^{5}$

\subsubsection{Criterios para la evaluación del léxico}

Para el proceso de evaluación del léxico de los diccionarios seleccionados nos basamos en los criterios de Fuentes et al., quienes resumen las recomendaciones en la literatura sobre dicha evaluación y consideran que los tres elementos siguientes son "ejes [...] en los que se puede organizar toda evaluación [de diccionarios] a) cantidad de datos lexicográficos, b) calidad de los datos lexicográficos [y] c) usabilidad del diccionario" (2005: 74).

\section{Selección de los diccionarios a evaluar}

\subsection{Selección de usuarios y diseño de la encuesta}

Una vez creado el marco evaluativo de la investigación, nuestro siguiente paso fue determinar cuáles diccionarios se evaluarían. Partiendo entonces de Frawley et al., quienes focalizan el papel del usuario en la creación de un diccionario y formulan preguntas clave como: ¿quién está utilizando este diccionario, para qué propósito y cómo? (2002: 12), consideramos necesario recabar su opinión para acotar la lista de diccionarios a evaluar. Desde luego, estamos conscientes de que existe una amplia gama de usuarios de diccionarios en lengua maya, por lo que nos limitamos a un grupo particular: 102 personas, entre ellos estudiantes nativohablantes y no-nativohablantes de la lengua maya en la península de Yucatán; estudiantes de maya como lengua extranjera en Estados Unidos y Europa, y maestros de lengua maya en la península de Yucatán, Estados Unidos y Europa, tanto nativohablantes como no-nativohablantes.

Cabe mencionar que, a la fecha, no existen diccionarios monolingüies en lengua maya; solamente se pueden encontrar diccionarios bilingües (principal-

5 Se trata la importancia de varios de estos elementos en el libro Making dictionaries: Preserving indigenous languages of the Americas, especialmente en Callaghan (2002), Frawley et al. (2002) y Hinton y Weigel (2002). 
mente en maya y español), por lo que los usuarios de los diccionarios existentes generalmente hablan más de una lengua. Por otro lado, resulta importante considerar que, dado que los diccionarios son textos escritos, sus usuarios saben leer y escribir en lengua maya y son, en su vasta mayoría, alfabetizados primero en español. Asimismo, el español resulta un requerimiento para alcanzar niveles más avanzados de escolarización, pues, hoy en día, solo algunas escuelas ofrecen enseñanza en la lengua maya y esto sucede únicamente para el nivel básico. Dada esta situación, decidimos diseñar una encuesta en SurveyMonkey, ${ }^{6}$ con preguntas en español e inglés, y no en lengua maya, ${ }^{7}$ con el fin de considerar usuarios tanto nacionales como extranjeros (véase el Anexo para un ejemplar de la encuesta). La encuesta aplicada fue en línea. Se envió una invitación vía correo electrónico a una lista de conocidos que estuvieran trabajando algún tópico relacionado con dicha lengua. Explicamos el objetivo de nuestra investigación y también solicitamos que la encuesta se difundiera entre más personas que trabajan con y en lengua maya, logrando una muestra basada en una red de conexiones en México y otros países. Consideramos, sin embargo, que una investigación a futuro podría abarcar un rango más amplio de usuarios.

Reconocemos que nuestra manera de difundir la encuesta y la elección de proveerla en ambas lenguas influyeron en el perfil de los participantes. Es decir, no pretendemos que nuestra muestra de encuestados sea representativa de todos los hablantes de lengua maya, sino del grupo más amplio de usuarios de diccionarios de lengua maya —individuos que hacen uso de la lengua y poseen la habilidad de leer y/o escribir. Este grupo también abarca a usuarios no-nativohablantes, quienes en su gran mayoría estudian y/o enseñan la lengua maya en instituciones de educación superior fuera de la República Mexicana.

Como se señala arriba, nuestro trabajo es exploratorio y tiene como propósito ser de utilidad para usuarios contemporáneos de diccionarios en lengua maya.

6 SurveyMonkey es un software gratuito para desarrollar encuestas en línea.

7 La encuesta no se diseñó en lengua maya porque: 1) quisimos recolectar datos de un público amplio de usuarios de diccionarios de lengua maya, 2) la alfabetización escolarizada se da primordialmente en lenguas dominantes, como el español y el inglés, pero aún es incipiente en lengua maya, y 3) existe variación escrita en la lengua maya. 
3.2. La selección de los diccionarios según los comentarios de los usuarios encuestados

Posteriormente, se enlistó un total de 11 diccionarios conocidos en la encuesta:

1) Diccionario maya popular: maya-español/español-maya (Academia de la Lengua Maya de Yucatán (ALMY), 2003);

2) Diccionario maya Cordemex (Barrera Vásquez, 1980);

3) Diccionario básico español/maya/español (Bastarrachea Manzano, Yah Pech \& Briceño Chel, 1992);

4) Maya for travelers and students: A guide to language and culture in Yucatan (Bevington, 1995);

5) A dictionary of the Maya language: As spoken in Hocabá, Yucatán (Bricker, Po'ot Yah \& Dzul de Po'ot, 1998); ${ }^{8}$

6) Diccionario introductorio español-maya, maya-español (Gómez Navarrete, 2009);

7) Tsolbe'enchuunil. Diccionario español-maya (Maglah Canul, 2002);

8) Diccionario maya: español-maya, maya-español (Martínez Huchim, 2008);

9) Maya (Yucatec) dictionary \& phrasebook: Maya-English/English-Maya (Montgomery, 2004);

10) Diccionario español-maya (Obon, 2009);

11) Diccionario de la lengua maya, Yucatán, Campeche y Quintana Roo. Alfabetización a población indígena (Secretaría de Educación Pública \& Instituto Nacional para la Educación de los Adultos, 2001).

Asimismo, solicitamos a los encuestados anotar cualquier otro diccionario que conocieran y que no estuviera en la lista. Mencionaron cinco diccionarios adicionales: el Calepino de Motul (Arzápalo Marín, 1995 [1877]), el Diccionario interactivo maya-español (Canché Moo, 2008), el Diccionario maya-náhuatl

8 Con el fin de incluir a los usuarios de maya angloparlantes, añadimos a la lista un diccionario bilingüe (Bricker, Po’ot Yah \& Dzul de Po’ot, 1998) y uno trilingüe (maya-inglés-español) (Montgomery, 2004). 
(Luján Castillo, 2014), el Diccionario de la conjugación de verbos en el maya yucateco actual (Yoshida, 2009) y el Vocabulario español-maya (Zavala \& Medina, 1898).

Con base en las respuestas de los usuarios, elegimos los diccionarios más utilizados. Para los diccionarios bilingües en maya-español e inglés-español, escogimos los textos consultados por un 50\% o más de los usuarios; en su mayoría, fueron empleados por más del 70\%. Esta selección nos brinda fiabilidad de datos, pero, más importante, nos brinda una perspectiva cabal del uso de los textos. Cabe aclarar que analizamos todas las respuestas de los encuestados y que únicamente nos limitamos durante el análisis al número total de diccionarios considerados. También incluimos un texto trilingüe, Montgomery (2004), como punto de comparación, ya que este texto y el de Bricker et al. (1998) ofrecen el ejemplo más completo para el análisis de macroestructura. Aunque más de un $50 \%$ de los usuarios mencionaron utilizar el Diccionario maya Cordemex, excluimos este diccionario de la investigación para enfocarnos únicamente en textos del maya contemporáneo. Con base en estos criterios, seleccionamos un total de seis diccionarios a evaluar: ALMY (2003), Bastarrachea Manzano et al. (1992), Bricker et al. (1998), Gómez Navarrete (2009), Martínez Huchim (2008) y Montgomery (2004).

\subsection{Selección de entradas de cada uno de los seis diccionarios seleccionados}

Para evaluar el léxico, seleccionamos una muestra de cada diccionario pues, dada la naturaleza exploratoria de esta investigación, no sería posible evaluar el total de las entradas. Para obtener la muestra, utilizamos la publicación $M a$ king dictionaries: A guide to lexicography and the Multi-Dictionary Formatter (Coward \& Grimes, 2000). Esta publicación sugiere 55 categorías semánticas, que abarcan tres categorías gramaticales: verbos, sustantivos y adjetivos. A partir de esta lista, cada uno de los tres autores sugirió una palabra para cada una de las 55 categorías semánticas. Esto resultó en una lista de 3 palabras por categoría. Posteriormente, escogimos una palabra muestra para cada categoría mediante consenso entre autores; en el 50\% de las veces, dos o tres autores sugirieron palabras iguales o muy similares (e. g., con las especificaciones de categoría gra- 
matical de sustantivo y la categoría semántica de agricultura, ${ }^{9}$ los autores eligieron kool (Rhodes), kool (Pomol Cahum), maíz (Chan Dzul)).

\section{Análisis de los diccionarios}

En esta sección consideramos las características generales de los diccionarios (páginas, formato del texto, cantidad de léxico, etcétera), el contenido (micro- y macro-estructuras), las fuentes y la presentación del léxico (ortografía y alfabeto utilizado, variante del maya utilizado y tratamiento de préstamos). Para cada uno de estos temas ofrecimos información de los diccionarios evaluados y, cuando fue relevante, también incluimos datos que proporcionaron los usuarios encuestados. Esta revisión cobra particular importancia por tratarse de una lengua indígena, debido a que la ortografía y el alfabeto de dichas lenguas están en proceso de estandarización, y es innegable su contacto con lenguas consideradas de mayor prestigio y dominio político y económico.

\subsection{Características generales, usos y usuarios de los diccionarios}

Cinco de los seis diccionarios fueron revisados en formato impreso y solo el diccionario de Gómez Navarrete (2009) en formato digital. Estos diccionarios presentan tamaños diferentes, lo que es importante al considerar la cantidad de léxico contenido en cada uno de ellos. Los diccionarios de Martínez Huchim (2008) y Montgomery (2004) tienen un tamaño aproximado de cuarta carta, mientras que los de Bastarrachea Manzano et al. (1992) y de la ALMY (2003), media carta. Por último, los diccionarios de Gómez Navarrete (2009) y Bricker et al. (1998) son los de mayor tamaño ( 17 x $23 \mathrm{~cm}$ en promedio). Cabe señalar que, en general, el tamaño de la tipografía de los diccionarios es de 10 puntos y que los diccionarios de Bastarrachea Manzano et al. (1992), la ALMY (2003) y Gómez Navarrete (2009) resaltan por el uso de mayúsculas para todas las entradas.

9 En la terminología de Coward y Grimes (2000), se utiliza Nagri para significar categoría gramatical y semántica, y se compone de: $N=$ 'noun', sustantivo en inglés, y agri = 'agriculture', agricultura en inglés. 
Cuatro de los diccionarios (ALMY, 2003; Bastarrachea Manzano et al., 1992; Gómez Navarrete, 2009, y Martínez Huchim, 2008) son bilingües, con secciones maya-español y viceversa. El diccionario de Bricker et al. (1998) es un diccionario bilingüe maya-inglés, sin la sección inversa. Y el de Montgomery (2004) es trilingüe, con dos secciones: maya-inglés-español e inglés-maya-español.

Las obras mencionadas están dirigidas a un público general (con la excepción de Bricker et al. (1998), que fue elaborado para estudiantes universitarios con conocimientos de lingüística). Sin embargo, quienes se interesan en los materiales de referencia en lengua maya poseen diversos perfiles y necesidades —nativohablantes de maya que buscan aprender a escribir en esta lengua, estudiantes de maya como L2, traductores, investigadores, entre otros- y configuran un público heterogéneo que no se acerca a los diccionarios con el mismo objetivo.

Relacionado con lo anterior, las respuestas de los usuarios encuestados indican que las razones más relevantes que los llevan a consultar los materiales referidos son como material de apoyo para:

1) preparar clases de/en lengua maya;

2) conocer el significado o equivalente de un término para traducciones;

3) complementar y verificar datos de investigación;

4) conocer el significado, el equivalente, la pronunciación y la forma escrita de alguna palabra, así como para conocer y saber utilizar la gramática de la lengua, tanto para nativohablantes como para los estudiantes de maya como segunda lengua, y

5) aclarar dudas sobre la escritura de una palabra, identificar posibles sinónimos, regionalismos o variantes a la hora de redactar en la lengua (sobre todo para mayahablantes interesados en la escritura).

La encuesta nos permite ver que la mayoría de los usuarios utilizan los diccionarios motivados por tópicos relacionados con la lengua maya; es decir, no se usan para aprender español u otra lengua que no sea el maya. En la encuesta también se trató el grado de satisfacción de los usuarios acerca de los materiales consultados (por ejemplo, el proceso de uso del diccionario, la organización de la información, la facilidad de búsqueda, etc.) y el grado de utilidad respecto 
al contenido (por ejemplo, la información contenida, la actualidad de los datos, lo adecuado de la información, la cantidad de ejemplos, etc.). Menos de $23 \%$ de los usuarios encuestados dijeron estar totalmente satisfechos con los diccionarios y menos de $14 \%$ los considera totalmente útiles. Surge entonces la interrogante de por qué un alto porcentaje de los encuestados hace notar su insatisfacción o la falta de utilidad del contenido y confección de los diccionarios. Discutimos estos aspectos en las secciones siguientes.

\subsection{Macroestructura de los diccionarios evaluados}

Entendemos por macroestructura de un diccionario la estructura general del texto, constituida por las partes en que el diccionario desarrolla su forma y por sus reglas sistemáticas de funcionamiento general, es decir, aquellos principios que rigen y condicionan el cuerpo de la obra (Pérez, 2005).$^{10} \mathrm{~A}$ grandes rasgos, la macroestructura puede abarcar información sobre el usuario destinatario del diccionario, el propósito de su publicación, cómo se creó y cómo se espera que se use. En el caso de la creación de diccionarios en lenguas indígenas, Callaghan (2002), Frawley et al. (2002) y Hinton y Weigel (2002) señalan la importancia de incluir esta información en el volumen (por ejemplo, indicar en un prefacio cómo usar el diccionario).

Sobre la macroestructura de los materiales analizados, encontramos una gran variación. Los diccionarios de Bastarrachea Manzano et al. (1992) y Martínez Huchim (2008) son los más básicos al incluir solamente el léxico maya-español, español-maya y una sección acerca del alfabeto usado. Por el contrario, los diccionarios de Montgomery (2004) y Bricker et al. (1998) sobresalieron por ser de los más completos. Montgomery (2004) incluye, además de las guías de pronunciación y gramática, una sección de frases básicas inglés-maya-español, algo que los usuarios, sobre todo estudiantes del maya, reportaron muy útil. Por su parte,

${ }^{10}$ La macroestructura incluye la ordenación del conjunto de los materiales que forman el cuerpo de un diccionario (por ejemplo, el orden alfabético), conjuntamente con el prólogo o prefacio, así como, a veces, una introducción fonética y gramatical, una guía de pronunciación, instrucciones para el usuario y los posibles anexos (glosario de nombres geográficos, listas de abreviaturas y siglas, glosario de nombres de pila, así como información sobre la civilización de determinados países) (Haensch \& Omeñaca, 2004). 
Bricker et al. (1998) incluyen una gramática del maya y un índice botánico. El hecho de que los dos diccionarios que utilizan el inglés como metalengua contengan macroestructuras más completas plantearía una investigación más allá de los alcances del presente trabajo, principalmente acerca de la posible correlación entre aspectos macroestructurales y la metalengua del diccionario. ¿Será que los autores de diccionarios asumen que los lectores en lengua española poseen un andamio sociocultural y lingüístico que les provee la información necesaria para usar sus textos y que haga innecesario incluir esta información en la macroestructura del diccionario? Nuestros datos demuestran que un $82 \%$ de los encuestados (en su mayoría hispanohablantes (84\%) y/o mayahablantes $(83 \%)$ ) prefieren una introducción gramatical a la lengua maya. En el Cuadro 2 se presenta una tabla resumen de las secciones contenidas en los diccionarios analizados.

CuADRo 2. Secciones analizadas como parte de la macroestructura de los diccionarios

\begin{tabular}{|c|c|c|c|c|c|c|}
\hline & $\begin{array}{l}\text { ALMY } \\
(2003)\end{array}$ & $\begin{array}{c}\text { Bastarrachea } \\
\text { Manzano et al. } \\
\text { (1998) }\end{array}$ & $\begin{array}{l}\text { Bricker } \\
\text { et al. } \\
\text { (1998) }\end{array}$ & $\begin{array}{c}\text { Gómez } \\
\text { Navarrete } \\
\text { (2009) }\end{array}$ & $\begin{array}{c}\text { Martínez } \\
\text { Huchim (2008) }\end{array}$ & $\begin{array}{c}\text { Montgomery } \\
\text { (2004) }\end{array}$ \\
\hline Índice & $x$ & $x$ & $\sqrt{ }$ & $x$ & $x$ & $\sqrt{ }$ \\
\hline Prefacio & $\sqrt{ }$ & $\sqrt{ }$ & $\sqrt{ }$ & $\sqrt{ }$ & $\sqrt{ }$ & $\sqrt{ }$ \\
\hline Guía de uso & $\sqrt{ }$ & $x$ & $\sqrt{ }$ & $x$ & $x$ & $\sqrt{ }$ \\
\hline Abreviaturas & $\sqrt{ }$ & $x$ & $\sqrt{ }$ & $\sqrt{ }$ & $\sqrt{ }$ & $\sqrt{ }$ \\
\hline Guía de pronunciación & $x$ & $x$ & $\sqrt{ }$ & $\sqrt{ }$ & $x$ & $\sqrt{ }$ \\
\hline Introducción gramatical & $x$ & $x$ & $\sqrt{ }$ & $x$ & $x$ & $\sqrt{ }$ \\
\hline Léxico & $\sqrt{ }$ & $\sqrt{ }$ & $\sqrt{ }$ & $\sqrt{ }$ & $\sqrt{ }$ & $\sqrt{ }$ \\
\hline Bibliografía & $\sqrt{ }$ & $x$ & $\sqrt{ }$ & $\sqrt{ }$ & $x$ & $x$ \\
\hline Otros & $\begin{array}{l}\text { Descripción } \\
\text { del alfabeto. }\end{array}$ & $\begin{array}{l}\text { Advertencia } \\
\text { sobre el uso } \\
\text { del alfabeto. }\end{array}$ & $\begin{array}{l}\text { Mapa de sitios } \\
\text { consultados. } \\
\text { Índice botánico. }\end{array}$ & $x$ & $\begin{array}{l}\text { Presentación } \\
\text { del alfabeto. } \\
\text { Inventario } \\
\text { fonémico de } \\
\text { consonantes. }\end{array}$ & $\begin{array}{c}\text { Guía } \\
\text { fraseológica. }\end{array}$ \\
\hline
\end{tabular}

\subsubsection{Cuantificación del léxico}

El número total de páginas, a pesar de ser un rasgo no homogéneo, puede dar una idea de la cantidad de léxico que integra cada uno de los diccionarios, ya que ninguno especifica el número total de entradas, subentradas, o sentidos. El diccio- 
nario con menos páginas fue el de Montgomery (2004), que destinó 37 de léxico maya-inglés-español y 48 de léxico inglés-maya-español. Los dos diccionarios con mayor número de páginas dedicadas al léxico son el de la ALMY (2003) (449, 271 maya-español y 178 español-maya) y el de Bricker et al. (1998) (319 páginas de léxico maya-inglés sin sección inversa) (véase el Cuadro 3). Sin embargo, reiteramos que la cantidad de léxico también es afectada por el tamaño de la tipografía, el formato (e.g., todas mayúsculas) y la cantidad de lenguas consideradas, entre otras cosas. Y, como señalamos arriba, es indispensable considerar la calidad del léxico junto con su cantidad.

CuAdro 3. Cantidad de páginas dedicadas al léxico en los diccionarios evaluados

\begin{tabular}{|c|c|c|c|c|c|}
\hline Diccionario & LÉxICO 1 & LÉxICO 2 & LÉXICO TOTAL & OTRA INFORMACIÓN & TOTAL dE PÁGINAS \\
\hline ALMY (2003) & $\begin{array}{c}\text { Maya-Español } \\
271\end{array}$ & $\begin{array}{c}\text { Español-Maya } \\
178\end{array}$ & 449 & 11 & 461 \\
\hline $\begin{array}{l}\text { Bastarrachea Manzano } \\
\text { et al. (1998) }\end{array}$ & $\begin{array}{c}\text { Español-Maya } \\
68\end{array}$ & $\begin{array}{l}\text { Maya-Español } \\
57\end{array}$ & 125 & 13 & 138 \\
\hline Bricker et al. (1998) & $\begin{array}{c}\text { Maya-Inglés } \\
319\end{array}$ & - & 319 & 113 & 410 \\
\hline Gómez Navarrete (2009) & $\begin{array}{c}\text { Español-Maya } \\
85\end{array}$ & $\begin{array}{l}\text { Maya-Español } \\
80\end{array}$ & 165 & 27 & 192 \\
\hline Martínez Huchim (2008) & $\begin{array}{c}\text { Español-Maya } \\
128\end{array}$ & $\begin{array}{c}\text { Maya-Español } \\
107\end{array}$ & 235 & 19 & 254 \\
\hline Montgomery (2004) & $\begin{array}{l}\text { Maya-Inglés-Es- } \\
\text { pañol } \\
37\end{array}$ & $\begin{array}{l}\text { Inglés-Maya-Es- } \\
\text { pañol } \\
48\end{array}$ & 85 & 117 & 204 \\
\hline
\end{tabular}

Respecto a la cantidad de léxico, los usuarios encuestados comentaron que los diccionarios son suficientemente útiles (43\%), mientras que otro $43 \%$ reportó que eran mínimamente útiles. Sin embargo, también expresaron la insuficiencia de entradas - no siempre encontraron la palabra que buscaban. Según las respuestas de la encuesta, este último punto guarda relación directa con el uso y tratamiento de préstamos en la lengua maya. En los diccionarios en lengua maya, 84\% de los encuestados querían encontrar ejemplos de uso cotidiano de las palabras y $51 \%$ pedían que solo se incluyeran palabras de uso común en la actualidad; $78 \%$ 
indagaban por información sobre los diversos significados de cada palabra; $76 \%$ buscaban variantes (regionalismos) y aclaraciones sobre su uso; $75 \%$ pedían que se insertaran préstamos que se usan en el maya cotidiano, mientras que solo $45 \%$ solicitaban la incorporación de neologismos (que no son de uso común) y, en contraste, $25 \%$ preferían que estos últimos no se incluyeran; $43 \%$ aspiraban por la inclusión de arcaísmos (que no son de uso común), mientras que 26\% preferían lo contrario. Como se discute en los siguientes apartados, los préstamos léxicos, especialmente los del español, no fueron incluidos en los diccionarios evaluados.

\subsubsection{Ortografía y alfabeto}

Iribarren señala que la "ortografía es una selección normativa de las posibilidades que tiene un sistema de escritura para escribir un idioma en particular de manera uniforme y estandarizada" (2005: 53). El conocimiento ortográfico de un idioma incluye además reglas de uso de signos de puntuación, uso de mayúsculas y minúsculas, uso de diacríticos, segmentación de unidades gráficas y cualquier otro tipo de marcadores gráficos que den indicaciones sobre su lectura o escritura. Es decir, las reglas de ortografía no se refieren exclusivamente a las reglas de correspondencia entre el inventario de grafemas y el inventario de fonemas de una lengua (Iribarren, 2005).

Cuando tratamos lenguas indígenas, muchas de las cuales aún están en vías de estandarización, no siempre hay una norma ortográfica reconocida. En la lengua maya, en particular, existe un alfabeto acordado en 1984 por diversas instituciones relacionadas con esta lengua. ${ }^{11}$ Aun así, hay muchos autores que prefieren escribir utilizando el alfabeto colonial que precede al alfabeto de 1984, y existen muchos textos que se publicaron con el alfabeto colonial — también la

${ }^{11}$ Las instituciones representadas eran el Instituto Nacional para la Educación de los Adultos (INEA), el Departamento de Educación Indígena (DEI), el Instituto Nacional Indigenista (INI), la Academia de la Lengua Maya de Yucatán (ALMY), el Instituto Nacional de Antropología e Historia (INAH) y la Unidad Regional de Culturas Populares (URCP). Aunque parece una representación amplia, Brody (2004) indica que no se incluyeron voces institucionales ni de individuos mayahablantes de los estados de Campeche o Quintana Roo (ni mucho menos de Belice, donde también se habla el maya yucateco), ni tampoco voces de mayahablantes del estado de Yucatán que no están afiliados a estas instituciones. Asimismo, argumenta que el alfabeto de 1984 no es representativo y que se formó por unos cuantos individuos para fines institucionales. 
mayoría de los apellidos, las toponimias y los señalamientos públicos usan este alfabeto. Sin embargo, hoy en día, la mayoría de los textos publicados en maya utilizan el alfabeto de 1984, aunque aún existe variación en el uso de los alfabetos y la ortografía.

Los diccionarios maya-español analizados se restringen al uso de las grafías del alfabeto maya acordado en 1984. Pero los diccionarios maya-inglés sí presentan diferencias; Montgomery (2004) difiere de los anteriores por el uso de las grafías $t z$ y $t z$, del alfabeto colonial, en lugar de la $t s$ y $t s^{\prime}$, del alfabeto de 1984 . Por otro lado, el diccionario de Bricker et al. (1998) destaca por el uso de un alfabeto fonético —el cual parece estar basado en el alfabeto fonético americanista (AFA). La variación en los alfabetos se aprecia en el Cuadro $4 .^{12}$

Cuadro 4. Alfabetos usados en los diccionarios analizados

\begin{tabular}{|c|c|}
\hline DicCIONARIO & Alfabeto \\
\hline ALMY (2003) & $a, b, c h, c^{\prime}, e, i, j, k, k^{\prime}, I, m, n, 0, p, p^{\prime}, r, s, t, t^{\prime}, t s, t s^{\prime}, u, w, x, y^{\prime}$ \\
\hline Bastarrachea Manzano et al. (1998) & $\begin{array}{l}\text { a, aa, áa, a', a’a, b, ch, ch', e, ee, ée, e', e’e, i, ii, ii, i', i'i, j, k, k', I, m, n, 0, o0, óo, o', } \\
\text { o’o, p, p', r, s, t, t', ts, ts', u, uu, úu, u', u’u, w, x, y' }\end{array}$ \\
\hline Bricker et al. (1998) & ?, b', ф, c', č, č', h, k, k', l, m, n, p, p', s, š, t, t', w, y \\
\hline Gómez Navarrete (2009) & $a, b, c h, h^{\prime}, e, i, j, k, k^{\prime}, l, m, n, 0, p, p^{\prime}, s, t, t^{\prime}, t s, s^{\prime}, u, w, x, y$ \\
\hline Martínez Huchim (2008) & $\begin{array}{l}\text { a, aa, áa, a', a’a, b, ch, ch', e, ee, ée, e', e’e, i, ii, íi, i', i’i, j, k, k', l, m, n, 0, o0, óo, o', } \\
\text { o'o, p, p', r, s, t, t', ts, ts', u, uu, úu, u', u’u, w, x, y' }\end{array}$ \\
\hline Montgomery (2004) & $a, b, c h, h^{\prime}, e, i, h, k^{\prime}, k^{\prime}, I, m, n, 0, p, p^{\prime}, r, s, t, t^{\prime}, t z, t z^{\prime}, u, w, x, y$ \\
\hline
\end{tabular}

${ }^{12}$ La variación en los alfabetos es indicio de una tensión con mucho precedente en la creación de diccionarios en lenguas indígenas (Callaghan, 2002; Frawley et al., 2002; Hinton \& Weigel, 2002; Pulte \& Feeling, 2002; Rice \& Saxon, 2002). La elección de un alfabeto fonético brinda la posibilidad de representar el rango de distinciones segmentales en lenguas indígenas, pero frecuentemente no son familiares para lectores no-lingüistas, mientras que los alfabetos arraigados a la tradición latina tienden a ser más reconocibles para lectores no especializados; en ambos casos, las cuestiones de orden alfabético pueden ser difíciles de reconciliar (e. g., con la representación de vocales, vocales con tono largo o alto, etc.) (Frawley et al., 2002: 8-9). 
Una gran mayoría de los encuestados (78\%) respondió que prefiere que el diccionario esté organizado en orden alfabético. Cabría investigar qué significa orden alfabético para los usuarios. Debido a la ausencia de estos datos, proveemos el ejemplo de un encuestado (1), cuya experiencia es análoga a los testimonios de otros participantes, que, al no estar familiarizado con la diversidad de alfabetos o convenciones ortográficas de los diccionarios en cuestión, experimentaba confusión al realizar una consulta:

(1) Los diccionarios que existen son utiles y la busqueda de palabra es razonablemente facil, sin embargo la variedad de ordenes "alfabéticos" (por ej. con respecto a las vocales cortas largas etc.) que se encuentra segun los diccionarios, o el hecho que no todos usan las mismas convenciones ortograficas puede volver dificultoso el proceso de encontrar palabras para los estudiantes o quienes no conocen las posibles variaciones. Las convenciones usadas por Bricker van bien para lingüistas pero no para maya hablantes ni para estudiantes principiantes. [Encuestado 97, 12 octubre 2015] ${ }^{13}$

Respecto a la ortografía de los diccionarios, los seis fueron publicados antes de que existiera alguna norma ortográfica para la lengua maya. En 2014, el Instituto Nacional de Lenguas Indígenas (Inali) publicó las Normas de escritura para la lengua maya (Briceño Chel \& Can Tec, 2014), las cuales recomiendan un alfabeto estándar y otras normas para la escritura de la lengua. Sin embargo, siendo una norma de reciente publicación para la lengua maya, su difusión aún se limita a ciertos sectores de lectoescritores en maya. Asimismo, suponemos que la adopción de la norma será variada, según varias ideologías lingüísticas (un concepto que elaboramos más adelante) y el hecho de que, como sus mismos autores enfatizan, es una norma y no un estándar.

\subsection{Microestructura de los diccionarios analizados}

La microestructura es la ordenación de todos los elementos que componen el artículo lexicográfico, y se ocupa de establecer los tópicos explicativos que funcionan sistemáticamente para cada uno de los artículos que integran el cor-

${ }^{13}$ Los testimonios de los encuestados se reproducen de manera textual. 
pus del diccionario (Pérez, 2005). Estos pueden ser, según el tipo de diccionario: enunciado del lema, indicaciones sobre la pronunciación, indicación de variantes gráficas, indicaciones morfológicas (e. g., conjugación de verbos, formación del plural), indicación de la categoría gramatical (sustantivo, verbo, etc.), indicación del género gramatical, diferentes clases de marcas que sirven para delimitar el uso de las unidades léxicas, definiciones y ejemplos de aplicación, entre otros (Haensch \& Omeñaca, 2004). Sobre la microestructura de los diccionarios evaluados consideramos las siguientes tres características: la especificación gramatical, las acotaciones y glosas, y los ejemplos, ya que estas resultaron ser las más importantes para los usuarios encuestados.

\subsubsection{Especificación gramatical}

Loos, Anderson, Day, Jordan y Wingate (2003) indican que una categoría gramatical es un conjunto de características sintácticas que expresan significados desde el mismo dominio conceptual, que ocurren en contraste el uno con el otro, y son típicamente expresados de la misma manera. Baker (2003) señala que diferentes idiomas tienen diferentes categorías léxicas, pero no hay una teoría sustantiva y generativa de dichas categorías, así que no es posible evaluar estos argumentos ni resolver estas controversias. Este tema es sumamente relevante a la hora de definir categorías gramaticales en lengua maya porque es una lengua que aún enfrenta debate sobre la definición de tales categorías, lo cual complica la tarea lexicográfica. Como una manera de mediar esta situación, Hinton y Weigel (2002: 163) recomiendan incluir un prefacio que explique cómo usar el diccionario, es decir, que provea de información macroestructural.

De acuerdo con estas limitaciones, basamos la evaluación de las categorías gramaticales en las preguntas que sugiere Haensch (2004: 135):

1) ¿Se dan las indicaciones gramaticales necesarias? Para estas se usan siglas llamadas marcas gramaticales.

2) ¿Se indica la categoría gramatical de las palabras como sustantivo, adjetivo, etc.? ${ }^{14}$

${ }^{14}$ Haensch incluye también una tercera pregunta a considerar para la evaluación de categorías 
Nuestra revisión reveló que no todos los diccionarios señalaban la categoría gramatical. Por ejemplo, las entradas en maya solo tenían tal indicación en Bricker et al. (1998), Gómez Navarrete (2009) y Montgomery (2004). Además, ningún diccionario indicó irregularidad gramatical, información necesaria para ciertos plurales (e. g., paal 'niño' y paalal 'niños') o formas verbales (e. g., xi’ik, subjuntivo del verbo bin 'ir'). Esto es especialmente importante en casos donde una entrada puede desempeñar varias funciones gramaticales, como la palabra míis, que puede significar 'escoba' (sustantivo) y 'barrer' (verbo).

Cabe destacar el diccionario de Martínez Huchim (2008), en el cual no se indica la categoría gramatical en ninguno de los léxicos. Los diccionarios de la ALMy (2003) y Bastarrachea Manzano et al. (1992) indican las categorías gramaticales en la sección español-maya, pero no en la sección maya-español. Caso contrario, los diccionarios de Bricker et al. (1998), Gómez Navarrete (2009) y Montgomery (2004) especifican la categoría gramatical de cada entrada, en cada sección (véase el Cuadro 5 a manera de ejemplo).

Además de la revisión sobre las indicaciones gramaticales, consideramos también si los diccionarios contenían una introducción gramatical para orientar a sus lectores en la comprensión de la gramática de las entradas léxicas y sobre las lenguas de cada una de las secciones de los diccionarios (maya, español o inglés). Solo dos de los seis diccionarios contenían una introducción gramatical del maya: Bricker et al. (1998) y Montgomery (2004).

Un $82 \%$ de los usuarios dijeron que la introducción gramatical de la lengua maya es una característica importante que buscan en los diccionarios. Aunado a lo anterior, 64\% de los usuarios buscaban una guía de pronunciación de la lengua maya, la cual solo incluyen los diccionarios de Bricker et al. (1998), Gómez Navarrete (2009) y Montgomery (2004).

gramaticales: “¿Se indica sistemáticamente el género de los sustantivos y la forma femenina de los adjetivos y especialmente de los sustantivos que denominan una profesión, actividad o actitud (nomina agentis)?" (2004: 135). Sin embargo, no consideramos esta pregunta en nuestra evaluación, ya que no existe género en la lengua maya. 


\subsubsection{Acotaciones y glosas}

Haensch (2004) señala que las acotaciones de las distintas acepciones de una palabra pueden hacerse mediante marcas lexicográficas (que pueden ser de especialidad, sobre la extensión geográfica del uso y estilísticas) o glosas (palabras o frases cortas aclaratorias no estandarizadas sobre el uso de la palabra, que aparecen en letra cursiva y entre paréntesis delante de cada acepción).

Ninguno de los diccionarios revisados utilizó un sistema regular de acotaciones o glosas. Es decir, no hay especificación alguna acerca de los contextos de uso o de los significados dados a cada una de las entradas, como puede observarse en el Cuadro 5.

Cuadro 5. Acotaciones y glosas utilizadas en la entrada para ts'oon/ tz'oon en los diccionarios analizados

\begin{tabular}{|c|c|c|}
\hline DICCIONARIO & ENTRADA MAYA-ESPAÑOL/INGLÉS & ENTRADA ESPAÑOL/INGLÉS-MAYA \\
\hline ALMY (2003) & $\begin{array}{l}\text { TS'00N 0: Escopeta.// Fusil.// Carabina.// Rifle: } \\
\text { tin kaxtaj junp'éel ts'oon: encontré un rifle.// } \\
\text { Cazar: ko'ox ts'oon kuuts: vamos a cazar pavo } \\
\text { de monte. }\end{array}$ & $\begin{array}{l}\text { ESCOPETA 0: Ts'oon. 4: (de recarga) but'bil } \\
\text { ts'oon. } \\
\text { CAZAR. v. 0: Ts'oon, p'uuj. }\end{array}$ \\
\hline $\begin{array}{l}\text { Bastarrachea Manzano } \\
\text { et al. (1998) }\end{array}$ & $\begin{array}{l}\text { TS'00N: Arma de fuego, escopeta, fusil, rifle; } \\
\text { cazar, fusilar. }\end{array}$ & $\begin{array}{l}\text { ARMA N/A } \\
\text { ESCOPETA f: ts'oon. } \\
\text { CAZAR v. ts'oon, p'uuj, chuuk. }\end{array}$ \\
\hline Bricker et al. (1998) & $\begin{array}{l}\text { (Debajo de ф'on T.) } \\
\text { c'òon } \\
\text { ф'òon, shoot, hunt; gun, rifle. cf. c'on. }\end{array}$ & -- \\
\hline Gómez Navarrete (2009) & $\begin{array}{l}\text { TS'00N.- sus. Cacería } \\
\text { TS'00N.- v.t. Cazar, fusilar, balear }\end{array}$ & $\begin{array}{l}\text { CACERÍA. sus. Ts'oon, p'uuj } \\
\text { CAZAR. v.t. Ts'oon. }\end{array}$ \\
\hline Martínez Huchim (2008) & $\begin{array}{l}\text { Ts'oon: arma de fuego.//Cazar, tirar con arma } \\
\text { de fuego.// Escopeta, fusil, rifle, fusilar. }\end{array}$ & $\begin{array}{l}\text { Cazar: chuuk, ch'úuk, p'uuj, ts'oon. } \\
\text { Escopeta: ts'oon. }\end{array}$ \\
\hline Montgomery (2004) & $\begin{array}{l}\text { tz'oon (tz'oohn) n. firearm, gun, shotgun; arma } \\
\text { de fuego, escopeta // tz'onik (TZ'OHN-eek) tr. } \\
\text { v. shoot; }\end{array}$ & $\begin{array}{l}\text { hunt; dispararlo, tirarlo; cazar // gun n. tz'oon } \\
\text { (tz'oohn); escopeta // firearm n. tz'oon (tz'oohn); } \\
\text { arma de fuego // shotgun n. tz'oon (tz'0ohn); } \\
\text { escopeta // hunt tr. v. tz'onik TZ'OHN-eek); cazar }\end{array}$ \\
\hline
\end{tabular}

Los ejemplos en el Cuadro 5 permiten apreciar la dificultad para encontrar el equivalente de las entradas, en tanto que no se advierte el cambio de categoría 
gramatical, ni se especifican los contextos o las posibles restricciones de uso. Aunado a esto, tampoco se proporcionan los ejemplos necesarios que ayuden a comprender el uso de la entrada en cuestión; así entonces, en el caso de la palabra ts'oon surgen dudas como: ¿significa 'escopeta', 'fusil', 'carabina', 'rifle' y 'arma de fuego' en todos los casos?, ¿hay diferencias morfosintácticas entre usar $t s^{\prime}$ 'oon como sustantivo y como verbo?, ¿en qué contexto $t$ 'oon significa 'cazar', 'fusilar' o 'disparar'?, entre otras.

\subsubsection{Ejemplos}

De acuerdo con Atkins y Rundell (2008), la naturaleza de los ejemplos varía conforme al tipo de diccionario y las necesidades y expectativas de los usuarios. Sin embargo, señalan que los ejemplos deberían ser: 1) naturales y típicos, 2) informativos y 3) inteligibles. Asimismo, estos autores indican que en un diccionario bilingüe los ejemplos complementan la información que ofrece cada entrada. Su propósito es ayudar a los hablantes de la lengua fuente a elegir el equivalente apropiado en la lengua meta y utilizarlo correctamente. Aunado a lo anterior, Bartholomew y Schoenhals (1983: 507) señalan que los ejemplos tienen varias funciones: 1) delimitar el significado de una entrada; 2) especificar el uso de la palabra en un contexto gramatical correcto; 3) distinguir entre sentidos aplicables y no-aplicables, y contextos de las glosas; 4) aclarar ambigüedades potenciales debidas a los múltiples significados de una glosa, y 5) presentar la cultura y el estilo literario locales.

Sobre el origen de los ejemplos, Bartholomew y Schoenhals (1983) indican que estos pueden provenir de hablantes no nativos, materiales traducidos, material escrito en lengua indígena o por nativohablantes; por cierto, sugieren no utilizar las dos primeras fuentes debido a la posibilidad de falta de naturalidad en las expresiones. Por su parte, Atkins y Rundell (2008) no recomiendan que se utilicen ejemplos tomados directamente de un corpus porque requieren demasiado espacio, pero sugieren el uso de colocaciones basadas en el corpus para componer ejemplos sencillos. Señalan que es mejor proveer ejemplos de contexto libre cuando sea posible.

Partiendo de este marco conceptual, realizamos una revisión de los ejemplos contenidos en los diccionarios evaluados. Encontramos que, en general, los 
diccionarios de Bastarrachea Manzano et al. (1992), Gómez Navarrete (2009), Martínez Huchim (2008) y Montgomery (2004) no proporcionan ejemplos de uso. Los diccionarios de la ALMY (2003) y Bricker et al. (1998) contienen ejemplos, pero carecen de un uso sistemático. Ofrecemos un ejemplo en el Cuadro 6.

Cuadro 6. Acotaciones y glosas utilizadas en la entrada para xímbal/xíinbal/ šímb’al en los diccionarios analizados

\begin{tabular}{|c|c|}
\hline DICCIONARIO & ENTRADA MaYa-ESPañol/InglÉS \\
\hline ALMY (2003) & $\begin{array}{l}\text { XílMBAL 0: Paseo: bin in ka'aj xímbal: voy a pasear.// Viaje.// Caminar: táan u xímbal } \\
\text { le chaambalo': el niño está caminando.// Visitar, hacer visitas: bin in ka'aj in xímbalt } \\
\text { in kiik: voy a visitar a mi hermana. }\end{array}$ \\
\hline Bastarrachea Manzano et al. (1998) & XÍlMBAL: Paseo, andanza; pasear, andar, caminar. \\
\hline Bricker et al. (1998) & $\begin{array}{l}\text { Debajo de ším X.) } \\
\text { šímb'al, n1. walk. } \\
\text { šímb'at, tv. visit } \\
\quad \text { táan in šímb'atik in kìik. I am visiting my older sister }\end{array}$ \\
\hline Gómez Navarrete (2009) & $\begin{array}{l}\text { XílNBAL. sus. Paseo. } \\
\text { X'IINBAL. v.i. Caminar, pasear }\end{array}$ \\
\hline Martínez Huchim (2008) & Xímbal: andanza, andar, caminar, pasear, paseo. \\
\hline Montgomery (2004) & $\begin{array}{l}\text { xímbal (SHÉEEM-bahl) in. v. go, walk; andar; caminar // go in. v. hóok'ol HÓOK’ohl), bin } \\
\text { (been), xímbal (SHÉEEM-bahl); salir; irse, andar // walk in. V. xíimbal (SHÉEEM-bahl); } \\
\text { caminar }\end{array}$ \\
\hline
\end{tabular}

Respecto a las fuentes de los ejemplos encontrados, Bricker et al. (1998) señalan que los ejemplos fueron elicitados, pero sin mayor especificación. Por su parte, el diccionario de la ALMY (2003) no indica el origen de los ejemplos, aunque se anota que para la obra en general se contó con el apoyo de redactores-informantes.

Un $83 \%$ de los usuarios encuestados comentaron acerca de la falta de ejemplos en los diccionarios y sugirieron la inclusión de ejemplos de uso cotidiano que permitan conocer los usos pragmáticos de las entradas. Ante la falta de ejemplos, muchos usuarios se han visto en la necesidad de consultar varias fuentes para aclarar sus dudas, tal como puede apreciarse en el comentario mostrado en (2), en el que el encuestado también da prueba de su evaluación sobre la naturalidad, información e inteligibilidad de los ejemplos: 
(2) En este sentido, puedo notar una diferencia entre Maya popular [ALMY, 2003] y Bricker. En general el contenido léxico de maya popular se acerca más al uso cotidiano que encuentro cuando estoy en Yucatán, aunque siempre hay ejemplos que no aparecen allí. El de Bricker se acerca menos al uso cotidiano pero sus ejemplos de cómo cada entrada léxica funciona gramaticalmente son excelentes. Uso una técnica doble para buscar palabras e inferir cómo se usan. Primero busco una traducción en el de Maya popular y luego busco la misma palabra (o raíz) en Bricker para ver cómo se usa esta palabra en contextos gramaticales diferentes. [Encuestado 90, 12 octubre 2015]

Entendemos que incluir ejemplos implica mayor extensión del diccionario y supone tiempo invertido por parte del lexicógrafo; sin embargo, resulta indispensable dado que su carencia limita la utilidad del diccionario para los usuarios, especialmente cuando las fuentes de comparación son escasas. También, siguiendo a Atkins y Rundell (2008) y Bartholomew y Schoenhals (1983), creemos que es importante solicitar ejemplos de hablantes nativos de la lengua para que estos sean naturales y típicos.

\subsection{Fuentes del léxico}

En este apartado consideramos importante el señalamiento de las fuentes del léxico de los diccionarios y la actualidad de los datos proporcionados. Existen diferentes fuentes para el léxico: otras obras lexicográficas, datos tomados de un corpus o de la lengua vernácula (por ejemplo, a través de la elicitación), entre otros.

Respecto a las fuentes del léxico en los diccionarios evaluados, encontramos que Bastarrachea Manzano et al. (1992), Martínez Huchim (2008) y Montgomery (2004) no proveen información sobre sus fuentes. Gómez Navarrete (2009) indicó que su diccionario se realizó a partir de una investigación bibliográfica y entrevistas en Chetumal, Quintana Roo, y la Universidad de Quintana Roo. En el diccionario de la ALMY (2003) se incluyeron las variantes correspondientes a cinco áreas geográficas: 1) área henequenera (Mérida y sus contornos), 2) zona del Camino Real (occidente de la península), 3) oriente del estado de Yucatán (Valladolid y sus contornos), 4) zona sur del estado de Yucatán y central del estado de Quintana Roo y 5) región de los Chenes en el estado de Campeche. Para 
cada una de las regiones se contemplaron a dos redactores-informantes, con excepción de la región de los Chenes, que contó con uno solo. Por último, para su diccionario, Bricker et al. (1998) desarrollaron un proceso muy elaborado: 1) generación de posibles raíces en maya, 2) glosado de las raíces válidas, 3 ) elicitación de las formas posibles por raíz, incluyendo raíces compuestas y ramas, 4) separación de homófonos y refinación de traducciones y 5) elicitación de ejemplos y edición. Las elicitaciones provienen de una sola comunidad: Hocabá, Yucatán, lo cual se indica en el título e introducción del diccionario.

Cabe destacar que, aunque la mitad de los diccionarios no indican sus fuentes, nuestra revisión revela que su léxico parece basarse en uno anterior. Por ejemplo, se observó que el contenido de los artículos del diccionario de Martínez Huchim (2008) se basa en los contenidos de los diccionarios de la ALMY (2003) y Bastarrachea Manzano et al. (1992). ${ }^{15}$ Esta práctica no es nueva, Hanks (2010) comenta que este método se usaba para obtener el corpus de vocabularios de la época colonial (creados principalmente por misioneros), los cuales, indica, demostraban quién leía a quién.

\subsubsection{Variante de maya utilizada}

Se consideró la variante de maya como factor para la evaluación debido a que en Yucatán existen diferentes registros (Berkley, 2001; Briceño Chel, 2002; Colazo-Simon, 2007; Cornejo Portugal \& Bellon Cárdenas, 2010; Cru, 2014; Gabbert, 2001; Guerrettaz, 2013, 2015; Hervik, 2003; Pfeiler, 1998; Pfeiler \& Hofling, 2006; Vrooman, 2000) y porque varios de los diccionarios evaluados utilizan alguna "variante" en específico.

En las obras analizadas encontramos puntos en común y puntos divergentes respecto a la variante de maya utilizada. En el Prefacio del diccionario de la ALMY (2003) se señala que observan "los fenómenos lingüísticos del maya actual", pues existe "la necesidad de un diccionario que diera cuenta del

${ }^{15}$ He aquí un ejemplo:

Gavilán: ch'úuy, éek'píip, i' (Martínez Huchim, 2008: 90).

GAVILÁN m. 0: Ch'úuy, i' 1: (conchero) Éek'píip (aLmy, 2003: 382).

GAVILÁN M, zool: Ch'úuy, i' (Bastarrachea Manzano et al., 1992: 37). 
maya moderno" (2003: s. p.). Por su parte, Bastarrachea Manzano et al. especifican que su diccionario "rescata el idioma maya hablado hoy en día [...] y en su uso actual" (1992: III) y precisan que esta obra se restringe tan solo al maya de Yucatán. Respecto a Gómez Navarrete (2009: 4), el autor señala que su obra es un diccionario bilingüe del léxico usual en maya. Por su parte, Martínez Huchim comenta en la Presentación de su diccionario que este "ofrece un vasto léxico del idioma usado en la actualidad en las diversas regiones de la Península yucateca, el cual se sustenta con terminologías del pasado" (2008: s. p.). Sin embargo, posteriormente, en el Prólogo a la tercera edición, indica que la "maya está viva y dinámica y, por lo mismo, cambiante. No es la de hace 200, ni 400 o 1200 años, sobre todo a nivel semántico" (2008: s. p.). Con las distinciones anteriores, no es claro qué variante del maya se incluye en el diccionario.

Montgomery (2004) asevera que su diccionario y guía fraseológica ofrece una selección de las palabras más comunes y frases útiles en lo que los lingüistas generalmente llaman maya yucateco, hablado virtualmente en todo Campeche, Quintana Roo y Yucatán. Finalmente, en Bricker et al. (1998) se señala que el léxico contenido corresponde al de un pueblo de la península de Yucatán: Hocabá.

\subsubsection{Tratamiento de préstamos en el léxico}

En nuestra revisión de los diccionarios encontramos tres estrategias para evitar la inclusión de préstamos: 1) la omisión, 2) la sustitución y 3) el uso de circunlocuciones. Estas estrategias no son hechos aislados, ya que investigadores como Pool Balam y Le Guen (2015) y Sobrino Gómez y Paz Ávila (2008) enmarcan dichos procesos dentro del fenómeno del purismo lingüístico.

Langer y Nesse (2012: 607) explican que existe gran variedad en las definiciones de purismo lingüístico; este puede abarcar desde los esfuerzos de purgar una lengua de cualquier elemento no deseado hasta únicamente eliminar los elementos extranjeros. Asimismo, señalan que los elementos considerados para su expulsión pueden variar ampliamente, incluyendo aspectos gramaticales, lexicales y otros. En el caso del maya yucateco, las estrategias puristas están altamente enfocadas en el nivel léxico — principalmente en la purga de elementos que provengan del español—, quizás porque es una de las dimensiones lingüísticas más accesibles al análisis para el usuario no especializado (Silverstein, 1981), 
aunque en el maya se puede encontrar influencia del español en otras dimensiones (por ejemplo, morfosintáctica, fonológica, semántica, pragmática, etcétera).

En gran parte, las tendencias puristas en el maya yucateco proceden de la creencia generalizada de que existen dos registros del maya: ${ }^{16}$ el xe'ek' maaya (variedad mezclada y corrompida por el español), que se contrapone a una variedad pura llamada jach maaya o maya pura o verdadera (Briceño Chel, 2002; Cru, 2014; Guerrettaz, 2013; Pfeiler \& Hofling, 2006; entre otros). A partir de esta creencia, existe un fuerte movimiento entre algunos sectores de mayahablantes que trabajan con la lengua, especialmente con la escritura y sus formas institucionalizadas, para purgarla de los préstamos del español.

La omisión es la primera estrategia que encontramos en los diccionarios para evitar la inclusión de préstamos. Se refiere al hecho de que la palabra simplemente no aparece en el texto. Esto presenta un gran problema para el usuario, que no tiene manera de saber si el ítem en cuestión se cubre con un préstamo o si el lexicógrafo fue negligente al excluir dicha expresión del diccionario (Bartholomew \& Schoenhals, 1983). Respecto a la omisión, notamos que en los diccionarios de la ALMY (2003), Bastarrachea Manzano et al. (1992), Gómez Navarrete (2009) y Martínez Huchim (2008) no se incluyeron préstamos, aunque fueran la única forma de uso cotidiano (véase el Cuadro 7); por ejemplo, no hay entradas para "liibro" (libro), "leeche" (leche) o "sáarten" (sartén). Los diccionarios de Montgomery (2004) y Bricker et al. (1998) incluyeron solo algunos préstamos; en este último hay un total de 156 préstamos con la indicación de su origen: español (la mayoría), mixe-zoque, náhuatl, totonaco, inglés, sayula-popoloca y zapoteco.

Bartholomew y Schoenhals (1983) indican que si los préstamos del habla común no se incluyen en un diccionario, la lista de vocabulario para esta lengua

${ }^{16}$ En cambio, Rhodes (2015a, 2015b, en revisión) argumenta que hay más de dos registros del maya yucateco, ya que identifica dos registros del jach maaya, el purista y el arcaico. También asevera que hay más registros lingüísticos del maya por documentar. Un registro lingüístico se define como un conjunto de prácticas linguiísticas que están asociadas con ciertos comportamientos o actividades sociales y las personas que participan en ellas (Agha, 1999; Halliday, 1978). No todos los hablantes de una lengua estarán familiarizados con todos sus registros lingüísticos, ni tendrán competencias iguales en los registros que sí manejan (Agha, 1999). (Véase también Rhodes, en revisión.) 
no está completa. Además señalan que es menos aceptable omitir una palabra común como lápiz que incluir una palabra relativamente rara que solo aparece en el habla de los bilingües, que manejan un rango más amplio de registros lingüísticos. También argumentan que, si los autores van a omitir préstamos, es necesario señalarlo y justificarlo en la introducción del diccionario. Con base en las recomendaciones de la literatura y de los usuarios encuestados, nos queda claro que los diccionarios evaluados dejan un gran vacío de información, por lo que quienes los consultan no siempre pueden resolver satisfactoriamente sus dudas. Resulta interesante que $75 \%$ de los encuestados sugirieron incluir préstamos que se usan en el maya cotidiano.

La segunda estrategia que se establece para evitar la inclusión de préstamos del español es su sustitución mediante arcaísmos (términos que han caído en desuso) y neologismos (vocablos de reciente creación). Sobre los primeros, Bartholomew y Schoenhals (1983) comentan que es importante señalar los que se incluyen en un diccionario (por ejemplo, mediante marcas como arc para arcaísmo). Concordamos con estos autores y opinamos que también es importante señalar los neologismos. Esta estrategia resulta práctica para investigadores de la lengua tanto como para hablantes que desean comunicarse en el ámbito vernáculo. Aunque, si atendemos las opiniones de los encuestados, se observa variación en los deseos de inclusión de estos términos: sí se deben incluir (45\% neologismos; $43 \%$ arcaísmos) o no (25\% neologismos; $26 \%$ arcaísmos). En los diccionarios que evaluamos no encontramos ejemplos de la sustitución de préstamos con neologismos. Sin embargo, esta práctica es muy común en los ámbitos académicos y en la enseñanza del maya, sobre todo con hablantes bilingües maya-español, quienes son conscientes del origen de los préstamos. Un buen ejemplo de esta práctica son las Normas de escritura para la lengua maya (Briceño Chel \& Can Tec, 2014).

La última estrategia para evitar el uso de préstamos es la circunlocución - la práctica de dar una explicación del objeto sin nombrarlo. Esta estrategia fue más notable en la sección español-maya de los textos de la ALMY (2003), Bastarrachea Manzano et al. (1992) y Martínez Huchim (2008). El hecho de encontrarse una explicación o definición en lugar de los equivalentes (préstamos) muestra una seria confusión entre las metodologías de elaboración de diccionarios bilingües y monolingües; pues, a decir de Kocjančič (2004), un diccionario 
monolingüe explica el significado o los significados de una entrada mediante las definiciones, mientras que un diccionario bilingüe principalmente ofrece equivalencias en la lengua de destino. Hinton y Weigel (2002: 164-165) señalan que, en el caso de los diccionarios bilingües, cada lengua se describe en los términos de la otra lengua; en el caso de la lengua subordinada, existe el peligro de la contaminación conceptual de la lengua superordinada. Notamos que la jerarquización de las lenguas en sí es un punto ideológico que merece reconocimiento.

Lara argumenta que, "desde el punto de vista lingüístico, ninguno de los vocablos perseguidos es perjudicial para una lengua; en la mayor parte de los casos la enriquece. Los diferentes tipos de purismo son siempre empobrecedores" (2006: 228-229). Además de lo anterior, Hagège (2009) argumenta que el purismo lingüístico crea las circunstancias favorables para la extinción de las lenguas (véase también Hill \& Hill, 1980, 1986). Y, para el caso particular del maya yucateco, Armstrong-Fumero (2009), Berkley (2001) y Guerrettaz (2013) señalan que la mayor influencia que tiene el movimiento purista en los hablantes monolingües del maya, que tienen generalmente bajos niveles de educación formal, es lo que nosotros llamamos un tipo de violencia psicológica y social que hace creer a los nativohablantes del maya que no saben hablar bien su lengua porque lo hacen con préstamos del español. Armstrong-Fumero (2009: 369) comenta que al encontrarse con el "maya puro" ("pure Maya"), algunas personas se sienten desempoderadas en relación con su lengua vernácula. Guerrettaz (2013: 235) describe cómo una maestra — una fuente de autoridad institucionalizada y estatal - posiciona a alumnos de la escuela primaria como no-expertos en su lengua natal e incompetentes en lengua maya por la utilización de palabras que originalmente provienen del español (por ejemplo: maamaj y papaj, derivados de mamá y papá). Berkley (1998: 19, 52; 2001: 351) explica que el hač Maya, que él relaciona con la lengua maya del pasado, evoca un pasado social y físicamente violento y tiende a silenciar a las mujeres y los jóvenes. Por último, ya que el hecho de hablar la lengua maya se toma ampliamente como marcador de pertenencia del grupo maya, opinamos que provocar que un nativohablante cuestione la autenticidad de su lengua no solo puede generar efectos psicológicos en el individuo en cuestión, sino también en la percepción (de sí mismo y de los demás) de su pertenencia en un grupo sociolingüístico y/o étnico. Sugerimos la necesidad de más investigación respecto a los efectos psicoló- 
gicos y sociales del purismo lingüístico, sobre todo el que proviene de fuentes institucionales.

Esto coincide con la realidad de la lengua maya y los diccionarios analizados, ya que, si bien esta lengua hoy en día incorpora préstamos, las obras lexicográficas que aquí se comentan los evitan — no obstante declaran su intención de representar el maya actual. Por lo tanto, estos diccionarios incumplen en cuanto a su representatividad y generan dudas considerables para sus usuarios. Para ilustrar los efectos que hemos explicado sobre el trato de los préstamos del español en los diccionarios en lengua maya, ofrecemos el ejemplo de la palabra amigo en el Cuadro 7.

CUADRo 7. La palabra amigo en los diccionarios evaluados

\begin{tabular}{|c|c|c|}
\hline DICCIONARIO & ENTRADA MAYA-ESPAÑOL/INGLÉS & ENTRADA ESPAÑOL/INGLÉSS-MAYA \\
\hline ALMY (2009) & (Sin entrada) & AMIG0, GA adj. 2: Uts. 3: Etail 5: Núup. \\
\hline Bastarrachea Manzano et al. (1998) & (Sin entrada) & $\begin{array}{l}\text { (Sin entrada para "amigo") } \\
\text { AMISTAD f: Uts bisbaj yéetel je' máaxake'. }\end{array}$ \\
\hline Bricker et al. (1998) & (Sin entrada) & (Sin sección inglés-maya) \\
\hline Gómez Navarrete (2009) & (Sin entrada) & $\begin{array}{l}\text { AMIGO. sus. Et ook, etail, éet bisbail, } \\
\text { etchi'li', etchi'il. }\end{array}$ \\
\hline Martínez Huchim (2008) & (Sin entrada) & AMIGO. etail, iich, núup. \\
\hline Montgomery (2004) & $\begin{array}{l}\text { áamigoh (áah-MEE-goh) n. friend; Sp. } \\
\text { Loanword; amigo }\end{array}$ & $\begin{array}{l}\text { friend n. áamigoh (áah-MEE-goh); Sp. } \\
\text { loanword; amigo }\end{array}$ \\
\hline
\end{tabular}

Como se puede apreciar en el Cuadro 7, los diccionarios utilizan la omisión, la sustitución, la circunlocución y el préstamo para tratar la palabra amigo. En el diccionario de Bastarrachea Manzano et al. (1992) no incluyen esta palabra, pero sí amistad, la cual definen por medio de una circunlocución. En los diccionarios de la ALmy (2003), Gómez Navarrete (2009) y Martínez Huchim (2008), los autores utilizan el arcaísmo (etail) y/o emplean la sustitución por redefinición semántica (núup, 'pareja', 'compañero'). Montgomery (2004) es el único diccionario que incluye una entrada para el préstamo: áamigoh; mientras que Bricker et al. (1998) tampoco insertan una entrada para el préstamo. Toda esta 
diversidad de información encontrada en los diccionarios evaluados es evidentemente confusa hasta para el usuario más especializado.

Además, los usuarios encuestados expusieron que los diccionarios ofrecen neologismos o arcaísmos como equivalentes en lugar de los préstamos (mayoritariamente del español) que utiliza la población en la actualidad. Esto trae consigo un problema de comunicación pues, al no ser términos de uso común, interrumpen la comunicación escrita y oral, tal como declara un encuestado en el comentario mostrado en (3).

(3) La mayoría de los diccionarios contienen arcaísmos y muchos neologismos, que ni la misma población usa. [Encuestado 56, 13 octubre 2015]

Como solución, un $83 \%$ de los usuarios sugirieron incluir léxico actual y cotidiano, y un $76 \%$, variantes (regionalismos). $75 \%$ de los encuestados pidieron incluir préstamos lingüísticos y señalaron algunas otras recomendaciones, como se puede observar en los comentarios (4a) y (4b).

(4) a. Para el diseño sería recomendable considerar la lengua maya que se habla en la actualidad y el significado que obtienen las palabras en los distintos contextos de uso. [Encuestado 48, 13 octubre 2015]

b. [Para] que un diccionario sea completo, las palabras aunque sean préstamos, que las incluyan. [Encuestado 48, 13 octubre 2015]

Un último punto que es necesario considerar respecto al trato de préstamos en los diccionarios es su ortografía. Aunque generalmente no es tan relevante la discusión sobre cómo representar los préstamos en textos escritos — dado que no se incluyen-, hay debate hoy en día entre las personas que se permiten usarlos (del español en lengua maya) acerca de cómo se deben escribir. La nuevas Normas de escritura para la lengua maya (Briceño Chel \& Can Tec, 2014) indican que deberían incluirse como se escriben en español y en cursivas, una postura que posiciona estas palabras como parte de otra lengua y no del maya. Sin embargo, Bartholomew y Schoenhals (1983) recomiendan escribir los préstamos según la lengua vernácula, porque los diccionarios en la lengua indígena crean 
el estándar para las lenguas que previamente no han sido escritas. Pool Balam y Le Guen (2015) ofrecen una perspectiva complementaria al argumentar que los préstamos del español son maya a través de procesos de mayanización, que pueden involucrar cambios fonológicos, morfológicos, sintácticos y semánticos. Estas recomendaciones hacen posible establecer un estándar para la escritura de los préstamos lingüísticos, en este caso en maya, y permiten al usuario reconocer la ortografía estándar de ambas lenguas, lo que contribuye al doble alfabetismo del hablante.

Finalmente, Bartholomew y Schoenhals (1983) recomiendan la adición de las letras necesarias de la lengua fuente a la lengua meta para hacer factible la representación escrita de los préstamos que pueden contener fonemas prestados de la lengua fuente. En el maya, por ejemplo, se usan las grafías $d, f$ y $g$ para representar muchos de los préstamos tomados del español, como documento, febrero y galleta, cuyos equivalentes en maya son dokumeento, febreero y gáayeta, respectivamente.

Estos últimos puntos sobre la ortografía de los préstamos en lengua maya y los efectos que podría tener para el alfabeto generan intensos debates sobre su trato en esta lengua. Sin embargo, reconocemos que existe una fuerte preferencia en los ámbitos institucionales hacia el purismo lingüístico, y esta preferencia podría acallar los debates o limitar la influencia de perspectivas alternativas.

Con todo lo descrito anteriormente se hacen evidentes las diversas posturas respecto a las variantes, registros, escritura y préstamos léxicos en la lengua maya. Se reconoce en los diccionarios evaluados, aunque principalmente de manera implícita, la existencia de variantes dialectales o regionales de la lengua maya, así como de variantes ideológicas (xe'ek' maaya versus jach maaya), sobre todo relacionadas con el uso de préstamos en el léxico. Acerca de esta última distinción, en la mayoría de los diccionarios evaluados los préstamos (que provienen principalmente del español) son excluidos mediante las diversas estrategias señaladas. Como consecuencia, los encuestados mencionaron sus efectos, tales como: la confusión ante la diversidad de propuestas de equivalencia o la carencia de estas. 


\section{Conclusiones}

Primera, cada diccionario es una representación de las ideologías lingüísticas de su(s) autor(es), es decir, el carácter situado, parcial e interesado de las concepciones y usos del lenguaje (Kroskrity, 2004: 496, basado en Errington, 2001: 110). Kroskrity explica además que estas concepciones, aunque articuladas de manera explícita o encarnadas (embodied) en la práctica comunicativa, representan esfuerzos incompletos o "parcialmente exitosos" para racionalizar el uso del lenguaje; típicamente, tales racionalizaciones son múltiples, dependientes del contexto y necesariamente construidas desde la experiencia sociocultural del hablante. En suma, las ideologías lingüísticas son creencias o sentimientos sobre el uso de los lenguajes en sus mundos sociales (Kroskrity, 2004: 498), ${ }^{17}$ es decir, informan las posturas y conocimientos de los autores respecto a las variantes, la ortografía y otros aspectos de sus textos. Es importante reconocer dicha aseveración al utilizar un diccionario con el fin de entender que este no representa la lengua de manera factual, sino que es una interpretación de una lengua en un momento particular y con base en ideologías lingüísticas particulares (no necesariamente explícitas). Ambas condiciones ocurren en el caso de los diccionarios bilingües porque representan dos mundos de conocimiento. Recomendamos que los creadores de los diccionarios describan claramente la metodología empleada para que los usuarios comprendan sus posturas teóricas e ideológicas y evalúen cómo usar la información obtenida de la obra. También sugerimos que, en la medida de lo posible, los autores consideren los efectos de sus decisiones e ideologías en la producción de sus textos; a pesar de que no siempre se podrán conocer los posibles efectos a priori, una colaboración cercana con comunidades de nativohablantes puede facilitar esta reflexión.

${ }^{17}$ La cita original es: "Language ideology, as succinctly defined by Errington [...] 'refers to the situated, partial, and interested character of conceptions and uses of language.' These conceptions, whether explicitly articulated or embodied in communicative practice, represent incomplete, or 'partially successful,' attempts to rationalize language usage; such rationalizations are typically multiple, context bound, and necessarily constructed from the sociocultural experience of the speaker" (Kroskrity, 2004: 496). "In sum, language ideologies are beliefs, or feelings, about languages as used in their social worlds" (Kroskrity, 2004: 498). 
Nuestra segunda recomendación, según las preferencias de los usuarios encuestados, es crear diccionarios que representen el uso cotidiano, lo cual requiere involucrar durante su elaboración a hablantes nativos de la lengua. Frawley et al. (2002: 12) afirman que, en contraste con diccionarios de lenguas occidentales, en la creación de diccionarios para lenguas indígenas de las Américas, la comunidad tiene un involucramiento activo y necesario en el desarrollo del diccionario. ${ }^{18}$ Junto con esta sugerencia, remarcamos la necesidad de formar lexicógrafos nativohablantes de la lengua maya. Varios autores que escriben sobre la creación de estas obras en lenguas indígenas indican que no todos los diccionarios son para todos los usuarios, y existen tensiones entre las necesidades de los linguiistas y las de los hablantes cotidianos de las lenguas (Frawley et al., 2002; Hinton \& Weigel, 2002). En particular, hay que reconocer que un diccionario ejerce poder y puede servir como una última entidad ratificante (Frawley et al., 2002: 14). ${ }^{19}$ Para la creación de diccionarios en lenguas en vías de estandarización esto es específicamente importante; las decisiones que se toman al describir una lengua nunca son neutrales (véase Hill, 2002; Irvine \& Gal, 2000), pero en el caso de estas lenguas, la influencia de dichas decisiones puede resultar particularmente autoritaria en contextos institucionalizados (como en la producción de un diccionario). Respecto a esto, cabe enfatizar que no existe una sola comunidad de mayahablantes, sino una gran diversidad; sin embargo, las instituciones de más influencia en la península y sus afiliados se alinean fuertemente a ideologías linguiísticas puristas. Por lo tanto, así como recomendamos la formación de lexicógrafos y lingüistas nativohablantes de la lengua maya, resulta imperativa una reflexión profunda acerca de las posturas ideológicas vis-à-vis la lengua y sus efectos.

Nuestra tercera recomendación refiere al deseo de los usuarios de que los diccionarios los ayuden a entender mejor el maya y a comunicarse en esta lengua.

${ }^{18}$ La cita original es: "In contrast [with dictionaries of Western languages], in American Indian-language dictionaries, the community has active and necessary involvement in the development of the dictionary" (Frawley et al., 2002: 12).

${ }^{19}$ La cita original es: "This is precisely because of the power that a dictionary can have over the community that no only is the source of the dictionary itself, but the ultimate ratifying body" (Frawley et al., 2002: 14). 
Para este fin, piden que los diccionarios representen el uso cotidiano. También solicitan una explicación de información gramatical, pronunciación y ejemplos reales y de uso habitual. En la revisión realizada, varios de los diccionarios declaran representar un maya actual, pero los usuarios no encuentran del todo útil la información contenida en ellos para fines comunicativos (1\% no útil, $43 \%$ mínimamente útil, $43 \%$ suficientemente útil, $14 \%$ totalmente útil); la falta de la inclusión de préstamos es un buen ejemplo de esto.

Los usuarios esperan encontrar en los diccionarios un modelo fiable a seguir para fines de escritura, así como ejemplos para las variantes de la lengua. En consecuencia, surgiría una fuerte discusión sobre si adoptar un solo estándar o representar las variaciones y, por ende, debería considerarse el objetivo de la obra misma (crear diccionarios: normativos, monolingües, históricos, de coloquialismos, de neologismos, etc.). No pretendemos tener la solución a esta cuestión, solo reconocemos que es una necesidad de los usuarios de diccionarios en lengua maya. Retomando la primera recomendación, una metodología explícita en el diccionario brinda a los usuarios mejor posibilidad de evaluar los textos que utilizan.

Finalmente, compartimos algunas consideraciones que surgieron de este estudio para futuras investigaciones sobre la creación de textos lexicográficos en lengua maya:

1) Un estudio comparativo que abarque diccionarios históricos y un mayor rango de diccionarios contemporáneos.

2) La creación de diccionarios monolingües en lengua maya.

3) Los efectos del purismo lingüístico más allá del texto, por ejemplo, en los usuarios.

4) La incorporación de los préstamos en la lengua maya vernácula y, consecuentemente, su posible incorporación en los textos lexicográficos.

5) La evolución y desarrollo de la ortografía en lengua maya.

\section{Referencias}

Academia de la Lengua Maya de Yucatán (almy) (2003). Diccionario maya popular: maya-español/español-maya. Mérida: Academia de la Lengua Maya de Yucatán. 
Agha, AsIF (1999). Register. Journal of Linguistic Anthropology, 9(1-2), 216-219.

Armstrong-Fumero, Fernando (2009). A heritage of ambiguity: The historical substrate of vernacular multiculturalism in Yucatán, Mexico [Un patrimonio de ambigüedad: el sustrato histórico del multiculturalismo vernáculo en Yucatán, México]. American Ethnologist, 36(2), 300-316.

Arzápalo Marín, Ramón (Ed.) (1995 [1877]). Calepino de Motul: diccionario maya-español. México: Universidad Nacional Autónoma de México.

Atkins, Beryl T. Sue, \& Rundell, Michael (2008). The Oxford guide to practical lexicography. Oxford: Oxford University Press.

BAKer, MARK C. (2003). Lexical categories: Verbs, nouns and adjectives. Cambridge: Cambridge University Press.

Barrera Vásquez, Alfredo (Dir.) (1980). Diccionario maya Cordemex. México: Ediciones Cordemex.

Bartholomew, Doris A., \& Schoenhals, Louise C. (1983). Bilingual dictionaries for indigenous languages. México: Instituto Lingüístico de Verano.

Bastarrachea Manzano, Juan Ramón; Yah Pech, Ermilo, \& Briceño Chel, Fidencio (1992). Diccionario básico español-maya-español. Mérida: Maldonado Editores.

Berkley, ANTHONy (2001). Respecting Maya language revitalization. Linguistics and Education, $12(3), 345-366$.

Bevington, GARY (1995). Maya for travelers and students: A guide to language and culture in Yucatan. Austin: University of Texas Press.

Briceño Chel, Fidencio (2002). Lengua e identidad entre los mayas de la península de Yucatán. En Los investigadores de la cultura maya (Vol. 10, tomo 2, pp. 370-379). Campeche: Universidad Autónoma de Campeche.

Briceño Chel, Fidencio, \& Can Tec, Gerónimo (2014). U nu'ukbesajil u ts'íibta'al maayat'aan/Normas de escritura para la lengua maya. México: Instituto Nacional de Lenguas Indígenas.

Bricker, Victoria; Po'ot Yah, Eleuterio, \& Dzul de Po'ot, Ofelia (1998). A dictionary of the Maya language: As spoken in Hocabá, Yucatán. Salt Lake City: University of Utah Press.

Brody, Michal (2004). The fixed word, the moving tongue: Variation in written Yucatec Maya and the meandering evolution toward unified norms (Tesis doctoral inédita). University of Texas, Austin. 
Callaghan, Catherine (2002). Writing a user-friendly dictionary. En William Frawley, Kenneth C. Hill \& Pamela Munro (Eds.), Making dictionaries: Preserving indigenous languages of the Americas (pp. 312-321). Berkeley: University of California Press.

CAnché Moo, Vicente (2008). Diccionario interactivo maya-español [multimedia]. México: Secretaría de Educación Pública.

Colazo-Simon, Antonia (2007). Les phénomènes glottaux en situation de contact linguistique: Maya et espagnol du Yucatán, Mexique (Tesis doctoral inédita). Université Sorbonne Nouvelle - Paris 3, París.

Cornejo Portugal, Inés, \& Bellon Cárdenas, Elizabeth (2010). "Cuando alguien habla la Maya se nota que son pobres": narrativas de identidad de los mayas yucatecos en torno a la radiodifusora indigenista La voz de los mayas, XEPET. Revista de Paz y Conflictos, 3, 6-22.

Coward, David F., \& Grimes, Charles E. (2000). Making dictionaries. A guide to lexicography and the Multi-Dictionary Formatter. Waxhaw: Summer Institute of Linguistics International.

CRU, JoseP (2014). From language revalorisation to language revitalisation?: Discourses of Maya language promotion in Yucatan (Tesis doctoral inédita). Newcastle University, Newcastle upon Tyne.

DebenPort, ERIN (2015). Fixing the books: Secrecy, literacy, and perfectibility in indigenous New Mexico. Santa Fe: School for Advanced Research Press.

Frawley, William; Hill, Kenneth C., \& Munro, Pamela (2002). Making a dictionary: Ten issues. En William Frawley, Kenneth C. Hill \& Pamela Munro (Eds.), Making dictionaries: Preserving indigenous languages of the Americas (pp. 1-22). Berkeley: University of California Press.

Fuentes Morán, María Teresa; García Palacios, Joaquín, \& Torres del Rey, Jesús (2005). Algunos apuntes sobre la evaluación de diccionarios. Revista de Lexicografía, 11, 69-80.

Gabbert, Wolfgang (2001). Social categories, ethnicity and the state in Yucatan, Mexico. Journal of Latin American Studies, 33(3), 459-484.

Gómez Navarrete, Javier Abelardo (2009). Diccionario introductorio español-maya, maya-español. Chetumal: Universidad de Quintana Roo.

Guerrettaz, Anne Marie (2013). Yucatec Maya teacher education and the struggle for a standard language (Tesis doctoral inédita). Indiana University, Bloomington.

Guerrettaz, Anne Marie (2015). Ownership of language in Yucatec Maya revitalization pedagogy. Anthropology \& Education Quarterly, 46(2), 167-185.

HAENSCH, GüNTHER (2004). La lexicografía bilingüe en la actualidad y su valoración. Revista de Lexicografía, 10, 133-138. 
Haensch, Günther, \& Omeñaca, Carlos (2004). Los diccionarios del español en el siglo xxi. Salamanca: Ediciones Universidad de Salamanca.

Hagège, Claude (2009). On the death and life of languages. New Haven: Yale University Press.

Halliday, Michael (1978). Language as social semiotic: The social interpretation of language and meaning. Baltimore: University Park Press.

Hanks, William (2010). Converting words: Maya in the age of the cross. Berkeley: University of California Press.

Hervik, Peter (2003). Mayan people within and beyond boundaries: Social categories and lived identity in the Yucatan. Nueva York: Routledge.

HiLl, JANE H. (2002). "Expert rhetorics" in advocacy for endangered languages: Who is listening, and what do they hear? [Retóricas expertas que abogan a favor de lenguas en peligro de extinción: ¿Quiénes están escuchando y qué es lo que escuchan?]. Journal of Linguistic Anthropology, 12(2), 119-133.

Hill, Jane, \& Hill, Kenneth (1980). Mixed grammar, purist grammar, and language attitudes in modern Náhuatl. Language in Society, 9(3), 321-348.

Hill, Jane, \& Hill, Kenneth (1986). Speaking Mexicano: Dynamics of syncretic language in Central Mexico. Tucson: University of Arizona Press.

Hinton, Leanne, \& Weigel, William F. (2002). A dictionary for whom? Tensions between academic and nonacademic functions of bilingual dictionaries. En William Frawley, Kenneth C. Hill \& Pamela Munro (Eds.), Making dictionaries: Preserving indigenous languages of the Americas (pp. 155-170). Berkeley: University of California Press.

Iribarren, Irene (2005). Ortografía española: bases históricas, lingüísticas y cognitivas. Caracas: El Nacional/Universidad Simón Bolívar.

Irvine, Judith, \& Gal, Susan (2000). Language ideology and linguistic differentiation. En Paul V. Kroskrity (Ed.), Regimes of languages: Ideologies, polities and identities (pp. 35-83). Santa Fe: School of American Research Press.

KocJAnČIČ, Polonca (2004). Acerca de la macroestructura y la microestructura en el diccionario bilingüe. Eslovenia: Universidad de Ljubljana. Recuperado de http://revije.ff.uni-lj.si/VerbaHispanica/article/viewFile/3705/3416

Kroskrity, Paul V. (2002). Language renewal and the technologies of literacy and postliteracy: Reflections from Western Mono. En William Frawley, Kenneth C. Hill \& Pamela Munro (Eds.), Making dictionaries: Preserving indigenous languages of the Americas (pp. 171-192). Berkeley: University of California Press. 
Kroskrity, Paul V. (2004). Language ideologies. En Alessandro Duranti (Ed.), A companion to linguistic anthropology (pp. 496-517). Malden: Blackwell.

Langer, Nils, \& Nesse, Agnete (2012). Linguistic purism. En Juan Manuel Hernández Campoy \& Juan Camilo Conde Silvestre (Eds.), The handbook of historical sociolinguistics (607-625). Malden: Wiley-Blackwell.

Lara, Luis Fernando (2006). Curso de lexicología. México: El Colegio de México.

Loos, Eugene E.; Anderson, Susan; Day, Jr., Dwight H.; Jordan, Paul C., \& Wingate, J. Douglas (Eds.) (2003). What is a grammatical category? En Summer Institute of Linguistics (SIL) International (Ed.), Glossary of linguistic terms. SIL International. Recuperado de https:// www.sil.org/resources/archives/1666

Luján Castillo, José Dimas (2008). Diccionario maya-náhuatl (aplicación de Google Play). Recuperado de https://play.google.com/store/apps/details?id=com.diccionario.maya.ockhamti. josedlujan\&hl=es

Maglah Canul, Gaspar (2002). Tsolbe'enchuunil. Diccionario español-maya (Manuscrito inédito). Quintana Roo.

Martínez Huchim, Ana Patricia (2008). Diccionario maya: español-maya, maya-español. Mérida: Dante.

Monterde Rey, Ana María (2007). Cómo hacer un diccionario [Power Point]. Las Palmas: Universidad de Las Palmas de Gran Canaria. Recuperado de https://studylib.es/doc/4661832/c\%C3\%B3mo-hacer-un-diccionario---universidad-de-las-palmas-de-...

Montgomery, John (2004). Maya (Yucatec) dictionary \& phrasebook: Maya-English, English-Maya. Nueva York: Hippocrene Books.

Obon, Pilar (2009). Diccionario español-maya. México: Época.

Pérez, Francisco Javier (2005). Pensar y hacer el diccionario. Nociones de lexicografía: definiciones, géneros, crítica e historia. Criterios para elaborar diccionarios, métodos de investigación y bibliografía lexicográfica. Caracas: El Nacional.

Pfeiler, BArbara (1998). El xe'ek' y la hach maya: cambio y futuro del maya ante la modernidad cultural en Yucatán. En Andreas Koechert \& Thomas Stolz (Eds.), Convergencia e individualidad: las lenguas mayas entre hispanización e indigenismo (pp. 125-140). Hanóver: Verlag für Ethnologie.

Pfeiler, Barbara, \& Hofling, Andrew (2006). Apuntes sobre la variación dialectal en el maya yucateco. Península, 1(1), 27-44.

Piotrowski, Tadeusz (1994). Problems in bilingual lexicography (Tesis doctoral inédita). Wydawnictwo Uniwersytetu Wrocławskiego, Breslavia. 
Pool Balam, Lorena, \& Le Guen, Olivier (2015). La integración de préstamos en maya yucateco. Una perspectiva interaccional acompañada de una reflexión sobre la ideología del lenguaje. Amerindia, 37(2), 343-384.

Pulte, William, \& Feeling, Durbin (2002). Morphology in Cherokee lexicography: The Cherokee-English dictionary. En William Frawley, Kenneth C. Hill \& Pamela Munro (Eds.), Making dictionaries: Preserving indigenous languages of the Americas (pp. 60-69). Berkeley: University of California Press.

Rhodes, CAtherine (noviembre, 2015a). Making Maya: Language standardization practices as sites for re-Mayanization in the Yucatán. Ponencia presentada en American Anthropological Association Meetings, Denver, Estados Unidos.

Rhodes, CATHerine (2015b). Making Maya linguistics: The production of Maya scientific expertise and models of personhood in the Yucatan today (Tesis doctoral inédita). University of Pennsylvania, Filadelfia.

Rhodes, CATHERINE (en revisión). The two registers of jach maaya: purist jach maaya and archaic jach maaya. Journal of Linguistic Anthropology.

Rice, Keren, \& Saxon, Leslie (2002). Issues of standardization and community in aboriginal language lexicography. En William Frawley, Kenneth C. Hill \& Pamela Munro (Eds.), Making dictionaries: Preserving indigenous languages of the Americas (pp. 125-154). Berkeley: University of California Press.

Secretaría de Educación Pública \& Instituto Nacional para la Educación de los AdulTos (2001). Diccionario de la lengua maya, Yucatán, Campeche y Quintana Roo. Alfabetización a población indígena. México: Instituto Nacional para la Educación de los Adultos.

Silverstein, Michael (1981). The limits of awareness. Sociolinguistic Working Paper, 84, $2-31$.

Sobrino Gómez, Martín, \& Paz Ávila, Lilia (2008). La condición actual de la lengua maya en Yucatán. Archipiélago, 16(60), 41-42. Recuperado de http://www.revistas.unam.mx/index. php/archipielago/article/viewFile/20030/19021

Vrooman, Michael (2000). The linguistic interdependence hypothesis and the language development of Yucatec Maya-Spanish bilingual children (Tesis doctoral inédita). University of Massachusetts, Amherst.

Yoshida, Shigeto (Comp.) (2009). Diccionario de la conjugación de verbos en el maya yucateco actual. Sendai: Tohoku University.

Zavala, Mauricio, \& Medina, Alejandro (1898). Vocabulario español-maya. Mérida: Imprenta de la Ermita. 
Anexo. Ejemplar de la encuesta a usuarios ${ }^{20}$

Usar diccionarios en lengua maya/ Using Maya language dictionaries

Introducción a la encuesta/ Introduction to this survey

Miguel Óscar Chan Dzul, Irma Pomol Cahum y Catherine Rhodes elaboramos esta encuesta para apoyar un trabajo lexicográfico que estamos realizando sobre el uso y el diseño de los diccionarios en lengua maya. Sus respuestas a esta encuesta son voluntarias y anónimas. Haremos un resumen de los resultados y este se presentará en el 2do Coloquio de la UNAM en la Península, titulado "El maaya t'aan: entre la variación y la estandarización" en noviembre de 2015 en Mérida, Yucatán. Si tienes alguna pregunta sobre la encuesta, favor de contactarnos en algunos de los siguientes correos electrónicos: mioscha7@hotmail.com,irma.pomol@gmail.com o rhodesc@gse.upenn.edu. Gracias por su tiempo y su apoyo con esta encuesta.

(Miguel Óscar Chan Dzul, Irma Pomol Cahum, and Catherine Rhodes elaborated this survey in support of a lexicographic study we are conducting about the use and design of Maya language dictionaries. Your responses to this survey are voluntary and anonymous. We will present a summary of the results of this survey at the 2 nd Colloquium of the UNAm on the Peninsula, entitled "El maaya t'aan: entre la variación y la estandarización" in Nov. 2015 in Merida, Yucatan. If you have any questions about this survey, please contact us at one of the following emails: mioscha7@hotmail.com, irma.pomol@gmail.com, or rhodesc@gse.upenn.edu. Thank you for your time and for your help with this survey.)

Información demográfica/ Demographic information

¿Cuál es tu nacionalidad?/What is your nationality?

Mexicana / Mexican

Estadounidense/American

Otra, favor de especificar/Other, please specify

${ }^{20}$ Adaptación de la encuesta realizada en línea. Se conservó toda la información requerida, solo se realizaron cambios de disposición para ajustarla al formato impreso de ELA (nota de los editores). 
¿Qué idiomas hablas? (Selecciona las que apliquen) / What languages do you speak? (Select all that apply)

- Maya

- Español/Spanish

Inglés/English

Otro(s), favor de especificar/Other(s), please specify

¿Eres maestro(a) de lengua maya? / Are you a Maya language teacher?
○ Sí/Yes
No

¿En qué institución(es) impartes clases de lengua maya? / At what institution(s) do you teach Maya language classes?

¿Eres alumno de lengua maya?/Are you a Maya language student?
Sí/Yes
No

¿Dónde tomas o has tomado clases de lengua maya?/Where do you take or have you taken Maya language classes?

¿Cuáles diccionarios de lengua maya usas? Selecciona todos los que usas./What Maya language dictionaries do you use? Select all of the ones that you use.

Diccionario Maya Popular: Maya-Español/Español-Maya (Academia de la Lengua Maya de Yucatán)

- Diccionario Básico Español/Maya/Español (Bastarrachea Manzano, Briceño Chel, Yah Pech) (cualquiera de sus ediciones / any of the editions)

- A Dictionary of the Maya Language as Spoken in Hocabá, Yucatán (Bricker, Po'ot Yah, Dzul de Po'ot)

Dictionary \& Phrasebook: Maya-English/English-Maya (Montgomery)

- Diccionario Español-Maya (Gaspar Maglah)

- Diccionario Introductorio Español-Maya/Maya-Español (Universidad de Quintana Roo, Gómez Navarrete)

\section{Diccionario Maya Cordemex}


Diccionario de bolsillo (pocket dictionary) de Dante

- Diccionario de la Lengua Maya/Maya Language Dictionary del Inea (Instituto Nacional para la Educación de los Adultos)

- Diccionario Español-Maya (Pilar Obón)

- Guía a la lengua maya para viajeros y estudiantes de Gary Bevington/Gary Bevington's Guide to Maya for Traveler's and Students

Si usas otros diccionarios en lengua maya, por favor, enlístalos aquí:/ If you use other Maya language dictionaries, please list them here:

\section{Principio del formulario}

Cuando usas un diccionario de lengua maya, ¿prefieres que sea monolingüe o bilingüe?/When you use a Maya language dictionary, do you prefer that it be monolingual or bilingual?
Monolingüe/
Bilingüe/bilingual
Depende/It depends
Monolingual

Si usas otros diccionarios en lengua maya, por favor, enlístalos aquí:/ If you use other Maya language dictionaries, please list them here:

¿En qué combinación de lenguas prefieres los diccionarios mayas bilingües?/What combination of languages do you prefer that a bilingual Maya language dictionary use?

Maya-Español/Spanish

- Maya-Inglés/English

- Ninguna preferencia, bilingüe con maya y cualquier de los otros idiomas que yo hablo/No preference, bilingual with Maya and any of the other languages that I speak

Maya con otra lengua, favor de especificar/Maya and another language, please specify: 
¿Qué factores son importantes para ti para adquirir un diccionario en lengua maya? (Selecciona todos los que aplican.)/What factors are important to you when you acquire a Maya language dictionary? (Select all that apply)

Que se pueda conseguir fácilmente/I can acquire it easily

Que sea económico/It doesn't cost much

Que los autores sean reconocidos/That the authors are well known

Que la editorial sea de renombre/That the publishing house is reputable

Que sea una edición impresa (libro)/That it have a print version (i.e. a book)

Que sea de fácil manejo/That it be easy to use

Que sea una edición electrónica (archivo digital)/That it be an electronic version

Que sea bilingüe/That it be bilingual

Si hay otras cosas que consideras a la hora de adquirir un diccionario en lengua maya, favor de enlistarlos aquí:/If there are other things you consider when you acquire a Maya language dictionary, please list them here:

¿Cuáles de los siguientes aspectos buscas en un diccionario en lengua maya? (Selecciona todos los que aplican)/Which of the following aspects do you look for in a Maya language dictionary? (Select all that apply)

Información para el usuario -información que indica para quién está diseñado el diccionario/Information for the user - information that indicates for whom the dictionary was designed

Información sobre cómo usar el diccionario/Information about how to use the dictionary

Una introducción gramatical a la lengua maya/A grammatical introduction to the Maya language

- Una guía de pronunciación en lengua maya/A Maya language pronunciation guide

- Información sobre cómo los autores escogieron el léxico (las palabras incluidas en el diccionario)/Information about how the authors chose the lexicon (the words included in the dictionary)

Información sobre abreviaturas y siglas/Information about abbreviations and acronyms

Ejemplos de uso cotidiano de las palabras/Examples of everyday uses of the words 
Ejemplos de las formas irregulares y excepciones de las palabras/Information about irregular forms and exceptions to words

- Información sobre los diversos significados de cada palabra/Information about the various uses of each word

Que esté organizado en orden alfabético/That it be organized in alphabetical order

Que esté organizado por tema o tópico/That it be organized by theme or topic

Que incluya variantes (regionalismos) y aclaraciones sobre su uso/That it include variations (regionalisms) and explanations about their uses

Que incluya préstamos (palabras que provienen de otras lenguas pero que se usan en el maya cotidiano)/That it include loan words (words that come from other languages but that are used in everyday Maya)

Que incluya neologismos (palabras de reciente creación que no son de uso común)/That it include neologisms (recently created words that are not commonly used)

Que NO incluya neologismos (palabras de reciente creación que no son de uso común)/That it NOT include neologisms (recently created words that are not commonly used)

Que incluya arcaísmos (palabras antiguas que no son de uso común)/That it include ancient words that are not commonly used

Que NO incluya arcaísmos (palabras antiguas que no son de uso común)/That it NOT include ancient words that are not commonly used

Que solo incluya palabras que son de uso común hoy en día/That it only include words that are commonly used today

Por favor, escribe cualquier otra característica que consideres importante en un diccionario de lengua maya: / Please list any other characteristics that are important to you in a Maya language dictionary:

¿Cuáles de los siguientes puntos consideras importantes en el diseño de un diccionario en lengua maya?/Which of the following points are important to you in the design of a Maya language dictionary?

El diccionario contiene suficiente información sobre la lengua maya (palabras, gramática, pronunciación, etc.)/The dictionary contains sufficient information about the Maya language (words, grammar, pronunciation, etc.) 
Puedo encontrar fácil y rápidamente la información que busco y que la contenga/I can easily and quickly find the information I'm looking for and the dictionary contains that information

La información sea inteligible (comprensible)/The information is understandable (comprehensible)

El diccionario provee suficiente información para que yo pueda valorar su fiabili$\mathrm{dad} /$ The dictionary provides sufficient information for me to evaluate its reliability El diccionario contiene información sobre el usuario para el cual fue diseñado/The dictionary contains information about the user for whom it was designed

Por favor, escribe algún otro aspecto que es importante para ti en el diseño de un diccionario en lengua maya/Please indicate any other aspects that are important to you in the design of a Maya language dictionary

¿Cuál o cuáles han sido las razones que te han motivado a usar un diccionario en lengua maya?/What reasons have motivated you to use a Maya language dictionary?

¿Cómo calificas tu nivel de satisfacción en el uso de diccionarios en lengua maya? Por satisfacción de uso referimos al proceso de usar el diccionario (ej. facilidad de búsqueda, organización de la información, etc.)/How would you qualify your level of satisfaction with using Maya language dictionaries? By satisfaction of use we are referring to the process of using a dictionary (e. g., easy of searching, organization of information, etc.)

\begin{tabular}{|c|c|c|c|}
\hline $\begin{array}{l}\text { No útil } \\
\text { (Not useful) }\end{array}$ & $\begin{array}{l}\text { Mínimamente } \\
\text { útil (Minimally } \\
\text { useful) }\end{array}$ & $\begin{array}{l}\text { Suficientemente } \\
\text { útil (Sufficient- } \\
\text { ly useful) }\end{array}$ & $\begin{array}{l}\text { Totalmente útil } \\
\text { (Completely } \\
\text { useful) }\end{array}$ \\
\hline
\end{tabular}

Por favor, explica tu respuesta:/Please explain your response:

¿Cómo calificas el nivel de utilidad de la información contenida en los diccionarios en lengua maya (ej. actualidad de los datos, información adecuada, suficientes ejemplos, etcétera)?/How would you qualify the level of utility of the information found in Maya language dictionaries (e.g., currentness of the entries, appropriate information, sufficient examples, etcetera)? 
No útil (Not useful)

\begin{tabular}{llll}
\hline Mínimamente & $\bigcirc$ & Suficientemente & Totalmente útil \\
útil (Minimally & útil (Sufficient- & (Completely \\
useful) & ly useful) & useful)
\end{tabular}

Por favor, explica tu respuesta:/ Please explain your response:

¿Has tenido algún problema en usar diccionarios de lengua maya? (Por favor, describe tu experiencia.)/Have you had any problems using a Maya language dictionary? (Please describe your experience.)

\section{Final del formulario}

¿Hay algo más que es importante para ti en el uso o el diseño de un diccionario en lengua maya? Favor de enlistarlo/Is there anything else that is important to you in the use or design of a Maya language dictionary? Please list it here 